\title{
TENT2, TUT4, and TUT7 selectively regulate miRNA sequence and abundance
}

Acong Yang ${ }^{1,3}$, Xavier Bofill-De Ros ${ }^{1,3}$, Ryan Stanton ${ }^{1}$, Tie-Juan Shao ${ }^{1,2}$, Patricia Villanueva ${ }^{1}$, Shuo $\mathrm{Gu}^{1}$

${ }^{1}$ RNA Mediated Gene Regulation Section; RNA Biology Laboratory, Center for Cancer Research, National Cancer Institute; Frederick, MD, 21702; United States.

${ }^{2}$ School of Basic Medicine, Zhejiang Chinese Medical University; Hangzhou, 310053; China

${ }^{3}$ These authors contributed equally

email: shuo.gu@nih.gov

\begin{abstract}
TENTs generate miRNA isoforms by 3' tailing. However, little is known about how tailing regulates miRNA function. Here, we generated isogenic HEK293T cell lines in which TENT2, TUT4 and TUT7 were knocked out individually or in combination. Together with rescue experiments, we characterized TENT-specific effects by deep sequencing, Northern blot and in vitro assays. We found that 3' tailing is not random but highly specific. In addition to its known adenylation, TENT2 contributes to guanylation and uridylation on mature miRNAs. TUT4 uridylates most miRNAs whereas TUT7 is dispensable. Removing adenylation has a marginal impact on miRNA levels. By contrast, abolishing uridylation leads to dysregulation of a set of miRNAs. Besides let-7, miR-181b and miR-222 are negatively regulated by TUT4/7 via distinct mechanisms while the miR-888 cluster is upregulated specifically by TUT7. Our results uncover the selective actions of TENTs in generating $3^{\prime}$ ' isomiRs and pave the way to investigate their functions.
\end{abstract}

[Keywords: miRNA; isomiRs; TENT; TENT2; TUT4; TUT7; uridylation; adenylation; miR-181; miR-222; miR-888 cluster] 


\section{Introduction}

MicroRNAs (miRNAs) are a class of regulatory noncoding RNAs. Despite their small size ( 22 $\mathrm{nt}$ ), they play critical roles in development and disease by regulating most, if not all protein-coding genes in mammals ${ }^{1}$. This highlights the importance of deciphering the regulation of miRNAs themselves.

After being transcribed from the genome, miRNAs are embedded in hairpin structures within primary transcripts known as pri-miRNAs. In many cases, multiple miRNAs are clustered within the same pri-miRNA, leading to coupled biogenesis. The miRNA-embedded hairpin is cropped from the pri-miRNA by the ribonuclease Drosha in the nucleus and then translocated to the cytoplasm. This hairpin, termed precursor miRNA (pre-miRNA), is further cleaved by Dicer, resulting in a small RNA duplex ${ }^{2}$. Depending on the end-thermostability, either the $5 p$ - or the $3 p$-arm of the pre-miRNA joins Argonaute proteins (AGO) to form an RNA-induced silencing complex (RISC), while the other miRNA strand is subsequently degraded ${ }^{3}$. Accordingly, mature miRNAs are designated as 5p- or 3p-miRNAs, depending on which strand is used. Once loaded into RISC, miRNAs recognize target mRNAs by sequence complementarity and repress their expression by mRNA degradation and/or inhibiting translation ${ }^{4}$.

Terminal nucleotidyltransferases (TENTs) modulate miRNA sequences by adding non-templated nucleotides to the $3^{\prime}$ end, a process referred to as tailing ${ }^{5-7}$. As a result, miRNA isoforms with heterogeneous 3' ends known as 3' isomiRs have been abundantly detected by deep sequencing 8,9 . Among 11 TENTs identified in mammals, TUT4 and TUT7 are implicated in uridylation of both precursor and mature miRNAs, whereas TENT2 is involved in adenylation $^{10,11}$. TENT-mediated modifications account for all isomiRs with non-templated tails and some of those with templated tails, the rest of which arise from alternative choices of cleavage sites by Drosha and Dicer during biogenesis ${ }^{12}$. The profile of 3' isomiRs is usually cell-type and tissue specific and can be used as a biomarker to identify many diseases, including cancer ${ }^{13-15}$, suggesting that isomiRs have a functional role. Indeed, case studies indicate that isomiRs can have distinct activities in a wide range of biological processes ${ }^{16-18}$. Recently, we demonstrated that 3' uridylation can alter the way miRNAs recognize targets ${ }^{19}$, providing one mechanism by which 3' isomiRs possess unique functions. Despite their importance, little is known about how 3' isomiRs are generated. It remains unclear whether different TENTs specifically modify various miRNA sequences. This becomes a major challenge in studying the biological function of $3^{\prime}$ isomiRs.

TENTs are known to regulate miRNA abundance via diverse mechanisms. TUT4 and TUT7 negatively regulate let-7 family members by oligo-uridylating their precursors in a LIN28-dependent manner ${ }^{20-22}$. The oligo-uridylated precursors are subsequently degraded by nuclease DIS3L2, leading to lower levels of mature let- $7^{23,24}$. On the other hand, TUT4, TUT7, and TENT2 positively regulate certain let-7 miRNAs by adding a single nucleotide to their 
pre-miRNAs in the absence of LIN28, promoting Dicer processing, which in turn leads to higher levels of mature miRNAs ${ }^{25}$. In Drosophila, uridylation negatively regulates the biogenesis of mirtrons ${ }^{26,27}$, a class of Drosha-independent miRNAs generated from introns. In addition, TENTs can modulate miRNA turnover by tailing the mature miRNA. In plants, uridylated miRNAs are removed by $3^{\prime}$ to $5^{\prime}$ exonuclease ${ }^{28,29}$. In mammals, TENT2-mediated adenylation stabilizes certain miRNAs in a cell context-dependent manner ${ }^{30,31}$ whereas adenylation of miR-21 by TENT4B leads to its decay ${ }^{32}$. In HEK293T cells, a subset of miRNAs including miR-222 are oligo-uridylated by TUT4 and TUT7 and subsequently degraded by DIS3L2 ${ }^{33}$. Finally, TUT4 and TUT7 regulate miRNA abundance by affecting the choice of strand selection. Uridylation of pre-miR-324 promotes the incorporation of its $3 p$ strand over the $5 p$ strand into downstream RISC in glioblastoma cells ${ }^{34}$. It remains to be examined whether additional miRNAs are regulated by TENTs.

Previous efforts studied adenylation and uridylation separately. TENT2, TUT4, and TUT7 and their homologues have been either knocked down or knocked out in various systems including human cells, mouse and C. elegans ${ }^{35-38}$. miRNAs were sequenced accordingly. However, different sequencing approaches were used, making it challenging to compare these datasets directly. Furthermore, rescue assays were missing in most of these studies, making it difficult to parse out the TENT-specific effects. Here, we systematically investigated how TENTs regulate miRNA sequence and abundance by generating a set of human HEK293T cell lines in which TENT2, TUT4, and TUT7 were knocked out individually or in combination. Deep sequencing analyses confirmed that uridylation or adenylation of miRNAs decreased accordingly, and could be rescued by ectopic expression of the corresponding tailing enzyme(s). Surprisingly, we found that TENT2 contributes to guanylation and uridylation in addition to adenylation on mature miRNAs in cells. Despite having comparable in vitro activity, TUT4 and TUT7 function differently in cells. TUT4 uridylates most miRNAs but nonetheless prefers miRNA substrates ending with guanosine. Uridylation mediated by TUT7, on the other hand, is apparently limited to the tailing of pre-miRNAs. While TENT2 specifically modulates miRNA sequences, removing TENT2 has only a marginal impact on miRNA levels in HEK293T cells. By contrast, abolishing uridylation led to specific up- and down-regulation of a set of miRNAs. In addition to let-7, we identified miR-181b-5p, miR-222-3p, and miR-888 cluster miRNAs that are regulated by TUT4/7-mediated uridylation via distinct mechanisms. Our results uncover the molecular basis for selective yet coordinated actions of TENTs, highlight the precise control of different 3' miRNA modifications in cells, and pave the way to investigate their functions.

\section{Results}

\section{miRNA 3' tailing is frequent and specific.}

To measure 3' tailing on mature miRNAs, we isolated endogenous AGO2 by immunoprecipitation (IP) in HEK293T cells and deep sequenced small RNAs in the pull-down. More than 7,000 3' isomiRs resulting from 683 miRNAs were consistently detected in two biological replicates (Supplementary Fig. 1a). We focused on isomiRs containing non-templated (NT) nucleotide(s) at the 3' end, which can only be generated by tailing. For each miRNA, we measured the percentage of trimmed and/or tailed by the relative abundance of the 
corresponding isomiRs. On average, more than $18 \%$ of miRNA reads had an NT tail (Fig. 1a), arguing that miRNA 3' tailing is not a minor event. As expected, NT tailing on 3p miRNAs was slightly more prevalent than that on $5 p$ miRNAs (Fig. 1a), attributed to tailings inherited from pre-miRNAs, which only affect $3 p$ miRNAs (Supplementary Fig. 1b). Nonetheless, a significant portion of tailing was observed on $5 p$ miRNAs, indicating that 3 ' tailing frequently occurs at mature miRNAs.

NT nucleotides added to the $3^{\prime}$ end of miRNA were not random but highly consistent between two biological replicates (Supplementary Fig. 1c). They were mainly $U$ and A, followed by a small portion $(\sim 10 \%)$ as $G$ and $<1 \%$ as $C$ (Fig. 1b). Most NT tails were short, with nearly half consisting of a single nucleotide. While mono- $U$ and mono-A were the most common NT tails, oligo-U and oligo-A tails were rare. Most oligo-tails (length $>1 \mathrm{nt}$ ) were composed of mixed nucleotides (Fig. 1c). The frequency of 3' NT tailing varied drastically among different miRNAs. While NT-tailed isomiRs were barely detectable for some miRNAs, they were the dominant form for others (Fig. 1d). For example, only $0.5 \%$ of miR-16-5p reads had an NT tail at the 3' end, whereas more than half of the miR-345-5p reads were NT-tailed (Fig. 1e), indicating that tailing is miRNA-specific. Together, these results suggest that miRNA 3' tailing is regulated in cells.

\section{Coordination of TENT2, TUT4, and TUT7 in miRNA 3' tailing.}

TENT2, TUT4, and TUT7 are the main TENTs implicated in miRNA tailing ${ }^{39}$. To comprehensively characterize their role in shaping miRNA sequence and abundance, we generated individual knockout, TUT4/TUT7 double knockout (DKO) and TENT2/TUT4/TUT7 triple knockout (TKO) cell lines by CRISPR. The expression of TENTs in WT cells and the depletion of TENTs in corresponding $\mathrm{KO}$ cells were confirmed by Western blot (Fig. 2A). We performed AGO2-IP and deep sequenced AGO-associated miRNAs in all KO cells. Uridylation and adenylation on the 200 most highly expressed miRNAs were measured by calculating the frequency of the corresponding nucleotide in the NT tails. As expected, depletion of TENT2 led to reduced adenylation, while depletion of TUT4 and TUT7 resulted in reduced uridylation for both $5 p$ and $3 p$ miRNAs (Fig. 2b and Supplementary Fig. 2a). Decreased uridylation was accompanied by a moderate increase in adenylation and vice versa, most likely due to improved accessibility of the miRNA 3' end when the competing TENT was absent. As a result, abolishing either TUT4/7-mediated uridylation or TENT2-mediated adenylation did not significantly diminish 3' tailing. Substantial reduction of 3' tailing and the resulting shortening of miRNA length was only observed in the TKO cells (Fig. 2b, c, Supplementary Fig. 2a, b). These results demonstrate that TENT2, TUT4, and TUT7 are the major TENTs that function coordinately in tailing mature miRNAs. Nonetheless, residual tailing observed in the TKO cells indicates that other TENTs, albeit to a lesser extent, also modify miRNA 3' ends.

Further analyses on several individual miRNAs confirmed the conclusions drawn from the global analysis (Supplementary Fig. 2c). We validated these results by Northern blot (Fig. 2d). The tailed isomiRs that are longer than the cognate miRNAs run slower during electrophoresis. Consistent with the sequencing result (Fig. 1e), miR-345-5p was extensively tailed. These tailed isomiRs were diminished only in the TKO cells (Fig. 2d, Supplementary Fig. 2c, d), suggesting 
that TENT2, TUT4, and TUT7 are responsible for tailing miR-345-5p. By contrast, depletion of TENT2, TUT4, and TUT7 had no impact on the tailing of miR-16-5p (Fig. 2d), the tailed isomiRs of which are primarily templated (Fig. 1e and Supplementary Fig. 2c) and most likely are a result of alternative Dicer cleavage during biogenesis. Tailed isomiRs of miR-24-3p, which are mainly uridylated, were affected more by the depletion of TUT4/7 than that of TENT2 (Supplementary Fig. 2c, d). Tailing on miR-24-3p was greatly reduced in the DKO cells (Fig. 2d), indicating that TENT2 cannot fully complement the tailing mediated by TUT4/7. This suggests that adenylation and uridylation at miRNA 3 ' ends are specific.

\section{TENT2 contributes to adenylation, uridylation, and guanylation of miRNAs.}

Our comprehensive set of knockout cells provided a unique opportunity to investigate TENT2-mediated miRNA tailing in vivo, which can be measured not only by comparing miRNAs between WT and TENT2-KO cells, but also by comparing the DKO and the TKO cells (Fig. 3a). With independent colonies serving as biological replicates, we were able to document consistent changes in miRNA tailing with high confidence. Furthermore, we performed the corresponding rescue assays with either a GFP control, WT TENT2 or catalytic-dead (CD) mutant in both TENT2-KO and TKO cells (Supplementary Fig. 3a). Endogenous miRNAs were deep sequenced accordingly. Together with the KO datasets, these results allowed us to identify TENT2-specific tailing events with or without TUT4/7 in the background (Fig. 3a).

First, we sought to investigate the nucleotide specificity of TENT2. To this end, we examined TENT2-mediated adenylation by analyzing highly expressed isomiRs with an NT mono-A tail. As expected, the relative percentage as well as the absolute abundance of the mono-adenylated isomiRs decreased upon TENT2 depletion while knocking out TUT4 and TUT7 had no impact (Fig. 3b, c). Expressing WT TENT2 but not the CD mutant in both TENT2-KO and TKO cells rescued the level of mono-adenylated isomiRs (Supplementary Fig. 3b, c). The abundance of isomiRs with a templated mono-A tail also changed accordingly, albeit to a lesser extent (Fig. $3 c$ ). This is consistent with the notion that only a portion of isomiRs bearing templated tails are a result of post-maturation modifications. IsomiRs adenylated with dinucleotide tails $(A A, A U$ and UA), although less abundant overall, followed a similar pattern of changes as mono-adenylated isomiRs (Supplementary Fig. 3d). Together, these results confirm the previously established adenylation activity of TENT2 on miRNA tailing, demonstrating the sensitivity and robustness of our approach.

We also examined the levels of uridylated isomiRs, which are not expected to be affected by TENT2. Consistent with previous studies, knocking out TENT2 in WT HEK293T cells had no impact on the level of mono-uridylated isomiRs (Fig. 3b, c). However, to our surprise, depleting TENT2 on top of TUT4/7 knockouts led to a consistent and significant reduction $(p=5.5 e-127)$ in uridylated isomiRs (Fig. 3b, c, comparing DKO with TKO). This suggests TENT2-mediated uridylation activity that is masked by TUT4 and TUT7 in WT cells and only becomes observable when TUT4 and TUT7 are absent. Given that TENT2 shows negligible uridylation activity in vitro $^{40}$, this effect observed in cells is unlikely to be mediated by TENT2 directly. Rather, TENT2 may recruit additional TENT(s) other than TUT4/7 to uridylate miRNAs. Supporting this idea, 
while isomiRs with a dinucleotide $A$ tail $(A A)$ or $U$ tail (UU) were dependent on TENT2 and TUT4/7 respectively, isomiRs with mixed dinucleotide tails (AU or UA) were more sensitive to the depletion of TENT2 but less dependent on TUT4/7 (Supplementary Fig. 3d). This uridylation effect of TENT2, while evident during TENT2 depletion, was not rescued by expressing either the WT or the CD mutant of TENT2 in TKO cells (Fig. 3c, Supplementary Fig. 3b). It is possible that transient expression of TENT2 failed to reconstitute the potential complex between TENT2 and other uridylation TENT(s) in cells.

Interestingly, the levels of NT mono-G-tailed isomiRs also decreased upon TENT2 depletion and could be rescued specifically by the WT TENT2, mirroring the pattern observed for mono-A-tailed isomiRs (Fig. 3b, c, Supplementary Fig. 3b, c). This is specific to guanylated isomiRs since isomiRs with a NT mono-C-tail were hardly detectable and were not responsive to TENT2 depletion and rescue (Supplementary Fig. 3e). To rule out the possibility that the observed G-tail was a misread of A due to artifacts introduced during library construction and/or Illumina sequencing, we deep sequenced a set of synthetic miRNAs and calculated the presumable $A$ to $\mathrm{G}$ conversion rate at both an internal position and at the last nucleotide. In both scenarios, the misread rate was below $0.1 \%$, which is in line with the known misread rate of Illumina platforms (Fig. 3d). Furthermore, this presumably $A$ to $G$ conversion was not observed for endogenous miRNAs at an internal position. Rather, it was specific to the NT tail, supporting TENT2-mediated guanylation in miRNA tailing.

\section{TENT2 selectively modifies mature miRNAs but has minimal impact on miRNA abundance.}

While TENT2 depletion had a global impact on miRNA adenylation, different miRNAs responded differently (Fig. 3b). For miRNAs such as miR-615-3p and miR-149-5p, the NT adenylated isomiRs were almost completely gone in TENT2-KO and TKO cells (Fig. 4a and Supplementary Fig. 4a), indicating that TENT2 is solely responsible for the adenylation of this set of miRNAs. On the other hand, depleting TENT2 in WT cells or DKO cells had marginal, if any impact on the adenylation of miRNAs such as miR-10a-5p (Fig. 4a), suggesting that these miRNAs were adenylated by TENT(s) other than TENT2. Supporting this idea, the level of the mono-A tailed isomiR of miR-21-5p, which is generated by TENT4B ${ }^{41}$, did not change after TENT2 depletion (Supplementary Fig. 4a). NT adenylated isomiRs of other miRNAs, for example let-7a-5p and miR-28-3p, were partially affected when TENT2 was knocked out (Fig. 4a, Supplementary Fig. 4a), indicating that both TENT2 and other TENT(s) contribute to their adenylation in HEK293T cells. In all scenarios, adenylated isomiRs increased in DKO cells, suggesting that TENT2 competes with TUT4/7 in accessing miRNA 3' ends. Supporting this idea, TENT2 rescue effect was greater in TKO cells where TUT4/7 were absent than that in TENT2-KO cells (Fig. 4a, Supplementary Fig. 4a).

To further analyze the miRNA specificity of TENT2, we calculated the fold change of each NT mono-A-tailed isomiR upon TENT2 manipulation. We used it as the measurement for how responsive the corresponding miRNA is to TENT2-mediated modifications. The "TENT2 sensitivity" for each miRNA was not random but consistent when TENT2 was depleted from two 
distinct genetic backgrounds (TENT2-KO vs. WT and TKO vs. DKO) (Fig. 4b). Furthermore, it was consistent between TENT2 depletion and rescue (Fig. 4c). As expected, a stronger correlation between TENT2 knockout and rescue was observed when the competing TUT4/7 were absent. Using a similar approach, we examined the miRNA-specificity of TENT2-mediated guanylation and uridylation. Supporting the idea that both G-tail and A-tail are mediated by TENT2 directly, miRNAs showed correlated sensitivity between adenylation and guanylation upon TENT2 depletion (Supplementary Fig. 4b). By contrast, there was no correlation between uridylation and adenylation, suggesting that the observed uridylation was mediated by TENT2 indirectly (Supplementary Fig. 4c). Finally, we measured miRNA specificity of TUT4/7-mediated uridylation (comparing DKO to WT cells) and found that it does not correlate with TENT2-mediated adenylation or uridylation (Fig. 4d), suggesting that the miRNA-specificity of TENT2 is unique and distinct from that of TUT4/7. Together, these results demonstrate that TENT2 is highly selective in tailing miRNAs.

Next, we sought to examine the impact of TENT2 on miRNA abundance by comparing miRNA levels in different KO cells. While depleting TENT2 in HEK293T cells reduced the adenylation of most miRNAs, only miR-873-5p, miR-222-3p, miR-138-5p and miR-34c-5p showed concurrent reduction in abundance (Fig. 4e). While this result supports the idea that these miRNAs are stabilized by TENT2-mediate adenylation, a mechanism established previously, further analyses indicate otherwise. Except miR-873-5p, these miRNAs showed a much smaller reduction when TENT2 was depleted in the DKO cells than when TENT2 was knocked out in WT cells (Fig. 4f), suggesting a potential role that TUT4/7 played in the observed reduction. It is possible that TENT2 depletion resulted in increased tailing by TUT4/7, which in turn led to miRNA decay. For miR-873-5p, the level of its passenger strand (miR-873-3p) was also reduced when TENT2 was abolished (Supplementary Fig. 4d). This suggests that TENT2 regulates miR-873 at a stage before the separation of guide and passenger strands, likely at the pri-miRNA level. Supporting this idea, TENT2 depletion also decreased the level of miR-876-5p (Supplementary Fig. 4e), which shares the same pri-miRNA transcript with miR-873. Furthermore, expressing TENT2 in either TENT2-KO or TKO cells failed to rescue the reduction of these miRNAs including miR-873 (Fig. 4g). Together, these results suggest that TENT2 has only marginal impacts on miRNA abundance in HEK293T cells.

\section{TUT4 but not TUT7 selectively uridylates most mature miRNAs.}

Applying a similar strategy, we investigated TUT4/7-mediated miRNA tailing by comparing DKO cells to WT cells as well as by comparing TKO cells to TENT2-KO cells (Supplementary Fig. 5a). Together with TUT4/7 rescue (Supplementary Fig. 5b), we were able to identify TUT4/7-specific effects. As expected, depleting TUT4 and TUT7 led to decreased levels of mono-U-tailed isomiRs (Supplementary Fig. 5c). Mirroring TENT2 depletion, knocking out TUT4 and TUT7 caused a subtle increase in the levels of mono-A-tailed isomiRs, demonstrating again the competition in accessing miRNA 3' ends between uridylating and adenylating TENTs (Supplementary Fig. 5c). Consistent with the idea that TENT2 also contributes to miRNA uridylation, knocking out TUT4/7 in the absence of TENT2 (TKO vs. TENT2-KO) had a much larger effect in reducing miRNA uridylation than the same procedure with TENT2 in the 
background (DKO vs. WT) (Supplementary Fig. 5d). Ectopically expressing both TUT4 and TUT7 from plasmids in the KO cells rescued miRNA uridylation but had no impact on adenylation (Supplementary Fig. 5e), indicating that, different from TENT2, TUT4/7 only exert uridylation activity in cells.

So far, we had evaluated the combined activity of TUT4 and TUT7. Taking advantage of TUT4-KO and TUT7-KO cells, we examined their activity individually. We analyzed the 100 most abundant uridylated isomiRs detected in these cells and calculated their relative abundance compared to that in the WT cells. To our surprise, while both TUT4 and TUT7 were expressed in HEK293T cells (Supplementary Fig. 5b), depleting TUT4 had a much bigger impact on miRNA uridylation than depleting TUT7 (Fig. 5a, Supplementary Fig. 5f). The absolute levels of uridylated isomiRs followed the same pattern (Fig. 5b). For most miRNAs, such as miR-126-5p, the level of mono-U-tailed isomiRs in TUT4-KO cells resembled that in DKO cells. Only a small portion of miRNAs (15 out of top 100 expressed, Supplementary Table 1) such as miR-148b-3p were affected by TUT7 depletion more than that by TUT4 depletion (Fig. 5c). Interestingly, 14 of these 15 miRNAs are $3 p$ miRNAs, the uridylation of which could be inherited from pre-miRNAs (Supplementary Fig. 1b). These results suggest that TUT4 is responsible for uridylation of mature miRNAs whereas TUT7-mediated uridylation is limited to a subset of miRNAs and potentially occurs only at the pre-miRNA stage. This is unlikely due to the inability of TUT7 to uridylate mature miRNAs, because ectopically expressing TUT7 in DKO or TKO cells had a similar, if not stronger effect in rescuing miRNA uridylation than expressing TUT4 (Supplementary Fig. 5g).

Next, we sought to examine the miRNA-specificity of TUT4 by identifying miRNAs whose uridylation was relatively more dependent on TUT4. To this end, we measured the fold change of the uridylation percentage for each miRNA upon TUT4 depletion in two comparisons (TUT4-KO vs. WT and DKO vs. TUT7-KO). The top 100 miRNAs with the biggest changes in uridylation in both scenarios were cataloged as "TUT4-sensitive" (Fig. 5d). Interestingly, G was enriched whereas $U$ and $C$ were disfavored at the last nucleotide position in these "TUT4-sensitive" miRNAs (Fig. 5e). We also probed the specificity of TUT4 by analyzing the rescue dataset. While ectopically expressing either TUT4 or TU7 rescued the loss of uridylation in both DKO and TKO cells, different miRNAs responded differently (Supplementary Fig. 5h), indicating they have distinct specificity. Once again, $G$ was enriched while $U$ and $C$ were disfavored as the ending nucleotide in the top 100 miRNAs that were consistently more responsive to TUT4 rescue than TUT7 rescue in both DKO and TKO cells (Supplementary Fig. $5 i)$. Together, these results suggest that the ending nucleotide plays a role in determining the miRNA specificity of TUT4.

To investigate the underlying mechanisms of these observations, we performed an in vitro uridylation assay. Mature let-7i (22 nt) was chemically synthesized, labeled with P32 at the 5' end and incubated with either TUT4 or TUT7. The products were separated on a PAGE gel. As expected, both TUT4 and TUT7 uridylated mature let-7i, resulting in isomiRs with U-tail, the length of which depended on the amount of enzyme and UTP/substrate ratio (Supplementary 
Fig. 5j). We performed in vitro uridylation assays on five miRNAs with the same amount of TUT4 and TUT7 proteins (Supplementary Fig. 5k). Regardless of the miRNA sequence, TUT4 showed more robust activity than TUT7 (Fig. 5f), partially explaining the superior activity of TUT4 observed in cells. However, miRNAs ending with a $G$ (miR-17-5p and miR-126-5p) were uridylated by TUT4 with similar efficiency as miRNAs ending with a $U$ (miR-26, let-7e, and let-7i) (Fig. 5f), despite the fact that only the former group was efficiently uridylated in cells. Furthermore, while mutating the last $G$ of miR-17-5p to a $C$ reduced its uridylation efficiency by TUT4 and TUT7, the same $G$ to $C$ mutation of miR-126-5p did not significantly affect its uridylation by either TUT4 or TUT7 (Fig. $5 \mathrm{~g}$ ). Together, these results show that TUT4's miRNA preference cannot be fully explained by the intrinsic feature of the enzyme, but is rather specific to miRNA tailing in cells.

\section{TUT4/7 regulate the abundance of a set of miRNAs via distinct mechanisms.}

Finally, we examined the impact of uridylation on miRNA abundance by comparing miRNA levels in KO cells to those in WT cells. Consistent with the model that TUT4 and TUT7 negatively regulate the biogenesis of let-7, we found let-7 family members among the most upregulated miRNAs in DKO and TKO cells (Fig. 6a). A previous study showed that a subclass of let-7 family members (let-7b, let-7d, let-7g, let-7i, miR-98) are more responsive to regulation via the LIN28-TUT-DIS3L2 machinery due to stronger binding between LIN28 and their precursors ${ }^{42}$. Only this subset but not the other let-7 family members were identified as the most upregulated miRNAs in DKO and TKO cells, demonstrating the robustness and specificity of our analysis. Several mirtrons including miR-1229-3p and miR-3620-3p were also upregulated, suggesting that TENT-mediated uridylation may regulate mirtron biogenesis in mammals as well. Consistent with our previous finding that mature miR-222-3p is targeted by the TUT-DIS3L2 machinery ${ }^{33}$, miR-222-3p abundance increased upon TUT4/7 depletion (Fig. $6 a)$.

TUT4 and TUT7 individual KO cells presented a relatively small number of differentially expressed miRNAs, while DKO cells had more and the TKO cells had the most (Supplementary Fig. 6a), a pattern similar to the observed effect of TENT-mediated uridylation on mature miRNAs (Fig. 5a). While this result supports that uridylation regulates miRNA abundance, the fact that both upregulated and downregulated miRNAs were observed in each $\mathrm{KO}$ cell line indicates that the underlying mechanisms are complicated. Indeed, further analysis on individual miRNAs showed no correlation between the changes in 3' mono-uridylation and the alteration in abundance upon TENT depletion (Supplementary Fig. 6b, c), indicating that 3' uridylation is unlikely a ubiquitous pathway for miRNA decay. Nonetheless, we were able to systematically identify miRNAs regulated by TUT4 and/or TUT7 by comparing miRNA abundance upon TUT4/7 knockout and the corresponding rescue (Fig. 6b). We focused on miRNA level changes that were consistently observed in DKO and TKO cells and could be rescued by ectopic expression of TUT4 and/or TUT7. Using this method, we identified a set of miRNAs that were either positively or negatively regulated by TUT4 and TUT7 in HEK293T cells with high confidence (Fig. 6c, Supplementary Table 1). 
Besides let-7 family members, our analysis revealed miR-181b-5p as one of the miRNAs negatively regulated by TUT4/7. We focused on miR-181b-5p due to its known function in cell proliferation and tumorigenesis ${ }^{43}$. We validated the sequencing results by Northern blot. Similar to let-7i-5p, let-7g-5p, and miR-222-3p, miR-181b-5p level was upregulated upon TUT4/7 depletion while the control miR-16-5p level remained unchanged (Fig. 6d). Furthermore, expressing TUT4, TUT7 or both, but not the GFP control in both DKO and TKO cells led to a specific downregulation (Fig. 6e). Interestingly, miR-181a-5p, a miRNA clustered with miR-181b-5p on the same pri-miRNA transcripts, followed a similar pattern of changes albeit to a smaller extent, suggesting that part of the regulation occurs at the pri-miRNA level. By contrast, miR-221-3p which is generated from the same pri-miRNA transcript as miR-222-3p, showed a distinctive pattern of changes, indicating that TUT4/7-mediated downregulation of miR-222-3p is post-transcriptional. Finally, to check if TUT4/7 regulate miR-222-3p and miR-181b-5p levels in the same way as let-7, we knocked down LIN28B, the LIN28 isoform expressed in HEK293T cells by siRNAs in WT, DKO and TKO cells (Fig. 6f). As expected, let-7g-5p was upregulated upon LIN28B knockdown in a TUT4/7-dependent manner. By contrast, neither miR-181b-5p nor miR-222-3p were affected by LIN28 knockdown with or without the presence of TUT4/7, indicating that their regulation is independent of LIN28B.

Surprisingly, we found that TUT4 and/or TUT7 positively regulate a set of miRNAs. However, most of these miRNAs were either lowly expressed or had only subtle changes upon TUT4/7 depletion and/or rescue. Unlike TUT4/7-mediated downregulation, the upregulation effect observed in deep sequencing could not be fully validated (Supplementary Fig. 6d). Nonetheless, we focused on those supported by Northern blot and found that miR-888-5p and miR-892a-3p were dramatically upregulated when TUT7 was ectopically expressed in either DKO or TKO cells (Supplementary Fig. 6e). Wild type TUT7, but not a CD mutant (TUT7-CD), resulted in the upregulation (Fig. 6g), indicating that uridylation activity is required. miR-888-5p and miR-892a-3p are two of seven miRNAs that reside within a human-specific cluster on chromosome $X$ (Supplementary Fig. $6 f)^{44}$. This strongly suggests that TUT7 led to the upregulation of the pri-miRNA transcripts. Supporting this idea, all seven miRNAs from the miR-888 cluster showed the same pattern of changes upon TUT4/7 overexpression (Supplementary Fig. 6g), indicating that TUT7-mediated uridylation either promotes the transcription or stabilizes the pri-miR-888 cluster transcript. Together, these results demonstrate that TUT4 and/or TUT7 regulate miRNAs including let-7, miR-222, miR-181b and miR-888 cluster by distinctive mechanisms.

\section{Discussion}

It has become apparent that 3 ' tailing is an integral mechanism in regulating miRNA function. A major challenge in studying miRNA tailing is the lack of understanding of the selective nature of TENT actions. Here, we used a genetic approach to tackle this question. Different from previous efforts, we generated a comprehensive set of single, double and triple $\mathrm{KO}$ cell lines, which enabled us to determine the functions of TENT2, TUT4 and TUT7 by at least two distinct genetic comparisons. Together with the rescue assays, we identified TENT-specific effects, 
some of which are too subtle to have been documented previously. We sequenced 38 samples, generating more than 180 million miRNA reads. Analyses of these sequencing results were further validated by Northern blots and in vitro assays. This comprehensive approach allows us to make novel findings that advance our understanding of how miRNAs are regulated by TENTs.

The role of TENT2 as an adenylation enzyme was well-established. Surprisingly, we found that human TENT2 also possesses guanylation activity in cells, which is consistent with a recent study where human TENT2 activity was measured by deep sequencing when tethered to a tRNA in yeast ${ }^{45}$. The resulting G-tail on miRNAs is unlikely an artifact because: 1) It was not observed on synthetic miRNA spike-in. 2) Knocking out TENT2, but not TUT4 and/or TUT7, led to the decrease of G-tail. 3) The reduction of G-tail in TENT2-KO cells was rescued by ectopically expressing the WT but not the CD mutant of the TENT2. Nonetheless, the guanylation activity of TENT2 is barely detectable in vitro ${ }^{40}$, suggesting that it could be enhanced by cellular cofactors. TENT4A and TENT4B, two adenylation enzymes, also have guanylation activity, which plays an important role in regulating mRNA turnover ${ }^{46}$. It is possible that this "mix-tailing" is a general feature of adenylating TENTs. The functional impact of TENT2 guanylation is yet to be determined. Another unexpected observation is that TENT2 also contributes to the uridylation of miRNAs. Since this effect is only observable when TUT4 and TUT7 are absent, it remains to be examined whether TENT2 mediates uridylation in WT cells or if it is only a compensation mechanism to maintain uridylation on miRNAs. Nonetheless, our results indicate that TENT2, in addition to TUT4/7, should be considered when investigating the function of uridylation on miRNAs.

Different from the nucleotide preference, little is known regarding the TENT preference towards various miRNA sequences in cells. Taking advantage of our comprehensive set of isogenic TENT KO cell lines as well as the corresponding rescue assays, we found that the 3' modifications mediated by TENT2, TUT4, and TUT7 are highly specific. Although TENT2-mediated adenylation and TUT4/7-mediated uridylation compensate each other to a certain extent, their specificity towards different miRNAs is distinctive and consistent among different KO cells. Given that TENT2 lacks an RNA binding domain, cellular cofactors such as QKI-7 likely account for its miRNA-specificity ${ }^{47,48}$. TUT4 and TUT7, on the other hand, can uridylate ssRNA in vitro without RNA-binding cofactors. Drosophila TUT enzyme Tailor prefers substrates ending with guanosine or uridine nucleotides in vitro ${ }^{27,49}$. Similarly, we found that human TUT4 and TUT7 uridylate G-ending and U-ending miRNAs with an efficiency higher than C-ending miRNAs in vitro, suggesting that this intrinsic feature of the TUT enzyme is conserved. However, consistent with a previous study ${ }^{50}$, their in-cell miRNA-specificity cannot be fully explained by their substrate preference measured in vitro. Together, these results suggest that 3' tailing on miRNAs is strictly regulated in cells. Given that the 3' end of a mature miRNA is inserted inside the PAZ domain of $A G O 2^{51}$, the accessibility of the $3^{\prime}$ end likely plays a role in regulating 3 ' tailing. It is possible that 3 ' modifications occur after miRNA pairing with its targets. In this way, the type of pairing and/or flanking sequences of the target site underlie the specificity of tailing on the corresponding miRNA. 
One of the best-characterized functions of TENT2, TUT4, and TUT7 is regulating miRNA abundance, which is highlighted by their prominent roles in the biogenesis of let-7 family members ${ }^{52}$. By contrast, whether or not miRNA tailing determines miRNA stability remains debatable. Consistent with several recent studies ${ }^{35,53,54}$, we found that the loss of uridylation or adenylation did not coincide with changes in miRNA abundance at a global scale. This suggests that 3' tailing does not play a general role in miRNA turnover. Rather, TENT2 stabilizes certain miRNAs in a context-dependent manner, while TUT4 and TUT7 regulate the abundance of a subset of miRNAs via multiple mechanisms. We found that TUT4 and TUT7 negatively regulate miR-222-3p but not the clustered miR-221-3p independently of LIN28B, indicating that the regulation likely occurs at the mature miRNA level. This is consistent with our previous observation that TUT4/7-mediated oligo-uridylation was specifically observed on miR-222-3p when DIS3L2 was depleted ${ }^{33}$. Together, these results support a model in which AGO2-associated mature miR-222-3p was targeted by the TUT-DIS3L2 machinery. Future studies are required to understand why miR-222-3p is specifically targeted in HEK293T cells. We also identified miR-181b-5p and miR-888 cluster miRNAs among others regulated by TUT4/7, indicating that the regulation is not limited to the biogenesis of let-7 family members. Nonetheless, the fact that only a small set of miRNAs are subjected to TENT regulation suggests that the biological functions of the 3' modifications may lie beyond controlling miRNA abundance.

TUT4 and TUT7 are thought to act redundantly. Our findings challenge this model by showing that TUT4 and TUT7 possess different tailing activities towards miRNAs. Depleting TUT4 had a major impact on miRNA uridylation overall, whereas the influence of knocking out TUT7 was limited to a subset of $3 p$ miRNAs. These results suggest that, while both contribute to the uridylation of pre-miRNAs, TUT4 is the major enzyme tailing mature miRNAs. We have previously found that TUT7 is more robust in oligo-uridylation of mature miRNAs, which are subsequently degraded by DIS3L $2^{33}$. These results suggest a model in which TUT4 is mainly responsible for mono-uridylation while TUT7 is involved in oligo-uridylation, leading to different outcomes: the former generates uridylated isomiRs, which might possess altered target repertoire ${ }^{19}$, while the latter results in degradation. In this way, cells distinguish these two pathways by functional specialization of TUT4 and TUT7. Supporting this idea, TUT7 but not TUT4 plays a major role in degrading the mRNAs of histone and $\mathrm{ZC} 3 \mathrm{H} 12 \mathrm{a}^{55,56}$. Furthermore, the upregulation of miR-888 cluster miRNAs, most likely due to the downregulation of unknown factors, is specific to TUT7. Further studies are required to understand the underlying mechanism for this functional specialization and how the specificity is achieved.

\section{Acknowledgments}

This work has been funded by the intramural research program of the National Cancer Institute, National Institutes of Health (ZIA BC 011566). The Seven Bridges Cancer Genomics Cloud has been funded in whole or in part with Federal funds from the National Cancer Institute, National Institutes of Health, Contract No. HHSN261201400008C and ID/IQ Agreement No. 17X146 under Contract No. HHSN261201500003I. 


\section{Contributions}

A.Y., X.B.-D.R. and S.G. designed the research. A.Y. performed all the experiments with help from R. S., TJ. S., and P.V.; X.B.-D.R. did all bioinformatic analyses; A.Y., X.B.-D.R., and S.G. analyzed the data. A.Y., X.B.-D.R. and S.G. wrote the paper.

\section{Competing interests}

The authors declare no competing interests.

\section{References}

1. Bartel, D. P. Metazoan MicroRNAs. Cell 173, 20-51 (2018).

2. Lee, Y., Jeon, K., Lee, J.-T., Kim, S. \& Kim, V. N. MicroRNA maturation: stepwise processing and subcellular localization. EMBO J. 21, 4663-4670 (2002).

3. Kawamata, T. \& Tomari, Y. Making RISC. Trends Biochem. Sci. 35, 368-376 (2010).

4. Gu, S. \& Kay, M. A. How do miRNAs mediate translational repression? Silence 1, 11 (2010).

5. Schmidt, M.-J. \& Norbury, C. J. Polyadenylation and beyond: emerging roles for noncanonical poly(A) polymerases. Wiley Interdiscip. Rev. RNA 1, 142-151 (2010).

6. Yu, S. \& Kim, V. N. A tale of non-canonical tails: gene regulation by post-transcriptional RNA tailing. Nat. Rev. Mol. Cell Biol. 21, 542-556 (2020).

7. Liudkovska, V. \& Dziembowski, A. Functions and mechanisms of RNA tailing by metazoan terminal nucleotidyltransferases. Wiley Interdiscip. Rev. RNA 12, e1622 (2021).

8. Wang, F., Johnson, N. R., Coruh, C. \& Axtell, M. J. Genome-wide analysis of single non-templated nucleotides in plant endogenous siRNAs and miRNAs. Nucleic Acids Res. 44, 7395-7405 (2016).

9. Chiang, H. R. et al. Mammalian microRNAs: experimental evaluation of novel and previously annotated genes. Genes Dev. 24, 992-1009 (2010).

10. Burroughs, A. M. et al. A comprehensive survey of 3' animal miRNA modification events and a possible role for 3' adenylation in modulating miRNA targeting effectiveness. Genome Res. 20, 1398-1410 (2010).

11. Wyman, S. K. et al. Post-transcriptional generation of miRNA variants by multiple nucleotidyl transferases contributes to miRNA transcriptome complexity. Genome Res. 21, 1450-1461 (2011).

12. Bofill-De Ros, X., Yang, A. \& Gu, S. IsomiRs: Expanding the miRNA repression toolbox beyond the seed. Biochim. Biophys. Acta Gene Regul. Mech. 1863, 194373 (2020).

13. McCall, M. N. et al. Toward the human cellular microRNAome. Genome Res. 27, 1769-1781 (2017).

14. Telonis, A. G. et al. Knowledge about the presence or absence of miRNA isoforms (isomiRs) can successfully discriminate amongst 32 TCGA cancer types. Nucleic Acids Res. 45, 2973-2985 (2017).

15. Bofill-De Ros, X. et al. Tumor IsomiR Encyclopedia (TIE): a pancancer database of miRNA isoforms. Bioinformatics (2021) doi:10.1093/bioinformatics/btab172.

16. Jones, M. R. et al. Zcchc11-dependent uridylation of microRNA directs cytokine expression. Nat. Cell Biol. 11, 1157-1163 (2009).

17. Yamane, D. et al. Differential hepatitis $C$ virus RNA target site selection and host factor activities of naturally occurring miR-122 3' variants. Nucleic Acids Res. 45, 4743-4755 
(2017).

18. $\mathrm{Yu}, \mathrm{F}$. et al. Naturally existing isoforms of miR-222 have distinct functions. Nucleic Acids Res. 45, 11371-11385 (2017).

19. Yang, A. et al. 3' Uridylation Confers miRNAs with Non-canonical Target Repertoires. Mol. Cell 75, 511-522.e4 (2019).

20. Heo, I. et al. Lin28 mediates the terminal uridylation of let-7 precursor MicroRNA. Mol. Cell 32, 276-284 (2008).

21. Hagan, J. P., Piskounova, E. \& Gregory, R. I. Lin28 recruits the TUTase Zcchc11 to inhibit let-7 maturation in mouse embryonic stem cells. Nat. Struct. Mol. Biol. 16, 1021-1025 (2009).

22. Thornton, J. E., Chang, H.-M., Piskounova, E. \& Gregory, R. I. Lin28-mediated control of let-7 microRNA expression by alternative TUTases Zcchc11 (TUT4) and Zcchc6 (TUT7). RNA 18, 1875-1885 (2012).

23. Chang, H.-M., Triboulet, R., Thornton, J. E. \& Gregory, R. I. A role for the Perlman syndrome exonuclease Dis3l2 in the Lin28-let-7 pathway. Nature 497, 244-248 (2013).

24. Faehnle, C. R., Walleshauser, J. \& Joshua-Tor, L. Mechanism of Dis3l2 substrate recognition in the Lin28-let-7 pathway. Nature 514, 252-256 (2014).

25. Heo, I. et al. Mono-uridylation of pre-microRNA as a key step in the biogenesis of group II let-7 microRNAs. Cell 151, 521-532 (2012).

26. Bortolamiol-Becet, D. et al. Selective Suppression of the Splicing-Mediated MicroRNA Pathway by the Terminal Uridyltransferase Tailor. Mol. Cell 59, 217-228 (2015).

27. Reimão-Pinto, M. M. et al. Uridylation of RNA hairpins by tailor confines the emergence of micrornas in drosophila. Mol. Cell 59, 203-216 (2015).

28. Tu, B. et al. Distinct and cooperative activities of HESO1 and URT1 nucleotidyl transferases in microRNA turnover in Arabidopsis. PLoS Genet. 11, e1005119 (2015).

29. Wang, X. et al. Synergistic and independent actions of multiple terminal nucleotidyl transferases in the 3' tailing of small RNAs in Arabidopsis. PLoS Genet. 11, e1005091 (2015).

30. Katoh, T. et al. Selective stabilization of mammalian microRNAs by 3' adenylation mediated by the cytoplasmic poly(A) polymerase GLD-2. Genes Dev. 23, 433-438 (2009).

31. D’Ambrogio, A., Gu, W., Udagawa, T., Mello, C. C. \& Richter, J. D. Specific miRNA stabilization by Gld2-catalyzed monoadenylation. Cell Rep. 2, 1537-1545 (2012).

32. Shukla, S., Bjerke, G. A., Muhlrad, D., Yi, R. \& Parker, R. The RNase PARN Controls the Levels of Specific miRNAs that Contribute to p53 Regulation. Mol. Cell 73, 1204-1216.e4 (2019).

33. Yang, A. et al. AGO-bound mature miRNAs are oligouridylated by TUTs and subsequently degraded by DIS3L2. Nat. Commun. 11, 2765 (2020).

34. Kim, H. et al. A Mechanism for microRNA Arm Switching Regulated by Uridylation. Mol. Cell 78, 1224-1236.e5 (2020).

35. Medhi, R. et al. RNA uridyl transferases TUT4/7 differentially regulate miRNA variants depending on the cancer cell-type. RNA (2021) doi:10.1261/rna.078976.121.

36. Mansur, F. et al. Gld2-catalyzed 3' monoadenylation of miRNAs in the hippocampus has no detectable effect on their stability or on animal behavior. RNA 22, 1492-1499 (2016).

37. Morgan, $M$. et al. mRNA 3' uridylation and poly $(A)$ tail length sculpt the mammalian maternal transcriptome. Nature 548, 347-351 (2017).

38. Le Pen, J. et al. Terminal uridylyltransferases target RNA viruses as part of the innate immune system. Nat. Struct. Mol. Biol. 25, 778-786 (2018).

39. Neilsen, C. T., Goodall, G. J. \& Bracken, C. P. IsomiRs--the overlooked repertoire in the 
dynamic microRNAome. Trends Genet. 28, 544-549 (2012).

40. Chung, C. Z., Jo, D. H. S. \& Heinemann, I. U. Nucleotide specificity of the human terminal nucleotidyltransferase Gld2 (TUT2). RNA 22, 1239-1249 (2016).

41. Boele, J. et al. PAPD5-mediated 3' adenylation and subsequent degradation of miR-21 is disrupted in proliferative disease. Proc Natl Acad Sci USA 111, 11467-11472 (2014).

42. Ustianenko, D. et al. LIN28 Selectively Modulates a Subclass of Let-7 MicroRNAs. Mol. Cell 71, 271-283.e5 (2018).

43. Indrieri, A., Carrella, S., Carotenuto, P., Banfi, S. \& Franco, B. The Pervasive Role of the miR-181 Family in Development, Neurodegeneration, and Cancer. Int. J. Mol. Sci. 21, (2020).

44. Hasegawa, T. et al. Characterization and Evidence of the miR-888 Cluster as a Novel Cancer Network in Prostate. Mol. Cancer Res. 16, 669-681 (2018).

45. Preston, M. A. et al. Unbiased screen of RNA tailing activities reveals a poly(UG) polymerase. Nat. Methods 16, 437-445 (2019).

46. Lim, J. et al. Mixed tailing by TENT4A and TENT4B shields mRNA from rapid deadenylation. Science 361, 701-704 (2018).

47. Chung, C. Z., Balasuriya, N., Siddika, T., Frederick, M. I. \& Heinemann, I. U. Gld2 activity and RNA specificity is dynamically regulated by phosphorylation and interaction with QKI-7. RNA Biol. 18, 397-408 (2021).

48. Hojo, $\mathrm{H}$. et al. The RNA-binding protein QKI-7 recruits the poly(A) polymerase GLD-2 for 3' adenylation and selective stabilization of microRNA-122. J. Biol. Chem. 295, 390-402 (2020).

49. Kroupova, A., Ivascu, A., Reimão-Pinto, M. M., Ameres, S. L. \& Jinek, M. Structural basis for acceptor RNA substrate selectivity of the 3' terminal uridylyl transferase Tailor. Nucleic Acids Res. 47, 1030-1042 (2019).

50. Thornton, J. E. et al. Selective microRNA uridylation by Zcchc6 (TUT7) and Zcchc11 (TUT4). Nucleic Acids Res. 42, 11777-11791 (2014).

51. Sheu-Gruttadauria, J. \& MacRae, I. J. Structural foundations of RNA silencing by argonaute. J. Mol. Biol. 429, 2619-2639 (2017).

52. Büssing, I., Slack, F. J. \& Grosshans, H. let-7 microRNAs in development, stem cells and cancer. Trends Mol. Med. 14, 400-409 (2008).

53. Vieux, K.-F. et al. Screening by deep sequencing reveals mediators of microRNA tailing in C. elegans. Nucleic Acids Res. 49, 11167-11180 (2021).

54. Morgan, M. et al. A programmed wave of uridylation-primed mRNA degradation is essential for meiotic progression and mammalian spermatogenesis. Cell Res. 29, 221-232 (2019).

55. Lackey, P. E., Welch, J. D. \& Marzluff, W. F. TUT7 catalyzes the uridylation of the 3' end for rapid degradation of histone mRNA. RNA 22, 1673-1688 (2016).

56. Lin, C.-C. et al. Terminal uridyltransferase 7 regulates TLR4-triggered inflammation by controlling Regnase-1 mRNA uridylation and degradation. Nat. Commun. 12, 3878 (2021).

57. Faehnle, C. R., Walleshauser, J. \& Joshua-Tor, L. Multi-domain utilization by TUT4 and TUT7 in control of let-7 biogenesis. Nat. Struct. Mol. Biol. 24, 658-665 (2017).

58. Bofill-De Ros, X. et al. QuagmiR: a cloud-based application for isomiR big data analytics. Bioinformatics 35, 1576-1578 (2019).

59. Kozomara, A., Birgaoanu, M. \& Griffiths-Jones, S. miRBase: from microRNA sequences to function. Nucleic Acids Res. 47, D155-D162 (2019). 


\section{Corresponding author}

Correspondence to Shuo Gu (shuo.gu@nih.gov).

\section{Methods \\ Plasmids}

For TENT2 gRNA expression vectors, 4 gRNAs were designed and synthesized, then cloned into Lenti-CRISPR-V2-puro at Bsmbl sites. TENT2 gRNA3-gRNA2/pTER+ plasmid was constructed by sequentially inserting the PCR amplified "U6-gRNA-sgRNA scaffold-TTTTTT" fragment into pTER+ at Bglll and Hindlll sites. The catalytic dead point mutations in pIRESneo-FLAG/HA-TENT2-CD (D212A and D214A) and pIRESneo-FLAG/HA-TUT7-CD (D1058A and D1060A) were introduced by mutagenesis ${ }^{31,57}$. All the oligo and primer sequences are listed in Table S2.

\section{Cell lines}

HEK293T cells were maintained in DMEM high glucose (Gibco) supplemented with 10\% heat-inactivated fetal bovine serum (Hyclone), $100 \mathrm{U} / \mathrm{ml}$ penicillin-streptomycin (Gibco) at $37^{\circ} \mathrm{C}$. TENT2-KO cells, TUT4-KO cells and TUT7-KO cells were generated by the lentiCRISPR V2 system using 4 corresponding gRNAs. TKO cells were generated by transfecting TENT2 gRNA3-gRNA2/pTER+ into DKO cells and selection with Zeocine. Plasmid transfections were performed using PolyJet ${ }^{\mathrm{TM}}$ DNA Transfection Reagent (SignaGen) according to the manufacturer's instructions. Lipofectermine RNAimax (Thermo scientific) was used for LIN28B siRNA (on-target smart pool, Dharmacon) transfections with a reverse transfection protocol.

\section{Northern blotting}

Total RNA was isolated from cells using Trizol (Life Technologies) and quantitated by Nanodrop. $20 \mu \mathrm{g}$ total RNA was run on $20 \%$ (w/v) acrylamide/8M urea gels with a ${ }^{32}$ P-labeled Decade marker (Ambion), and then transferred onto Hybond-N membranes (Amersham Pharmacia Biotech). After transfer, the membrane was either UV crosslinked or EDC-mediated chemical cross-linking (Sigma) was used. ${ }^{32} \mathrm{P}$-labeled probes that reverse complement to the targeted miRNAs were hybridized with membrane in PerfectHyb ${ }^{\text {TM }}$ Plus Hybridization Buffer (Sigma) overnight at $37^{\circ} \mathrm{C}$. After washing with $2 \mathrm{X}$ SSC with $0.1 \%$ SDS buffer for $3 \times 15$ min at $37^{\circ} \mathrm{C}$, the membrane was exposed to an Imaging Screen-K (Bio-Rad) overnight. Images were then analyzed by Typhoon Trio Imaging System (GE Healthcare). NB results were processed and quantitated by Image $\mathrm{J}$ software.

\section{Immunoprecipitation for endogenous AGO2 associated miRNAs}

One $10 \mathrm{~cm}$ dish of each cell line was lysed in $1 \mathrm{ml}$ modRIPA buffer (10 mM Tris-cl pH 7.0, 150 $\mathrm{mM} \mathrm{NaCl}, 1 \mathrm{mM}$ EDTA, $1 \%$ Triton $\mathrm{X}-100$, and $0.1 \% \mathrm{SDS}$ ) supplemented with proteinase inhibitors cocktail (Roche). Cell lysate was incubated with $50 \mu$ l SureBeads Protein G Magnetic Beads (Bio-Rad) plus $5 \mu \mathrm{g}$ mouse anti-AGO2 Monoclonal antibody at $4^{\circ} \mathrm{C}$ overnight with rotation. After washing 5 times with TBS buffer ( $50 \mathrm{mM}$ Tris- $\mathrm{HCl}[\mathrm{pH} 7.4], 150 \mathrm{mM} \mathrm{NaCl}$ ) at room temperature, the beads were lysed in $1 \mathrm{ml}$ Trizol (Life Technologies) for RNA extraction. 


\section{Western blotting}

Cells were lysed in modRIPA buffer with protease inhibitor cocktail (Roche). The cell lysates were quantitated using a BCA kit (Pierce) in the Glomax multi+ machine. $30 \mu \mathrm{g}$ protein of whole cell lysate or variable volume of Flag-IP samples was loaded into 4-20\% Mini-PROTEAN® TGX Stain-Free ${ }^{\mathrm{TM}}$ Gels (Bio-Rad) and then transferred onto a PVDF membrane using the Trans-Blot Turbo Transfer System (Bio-Rad). Primary antibodies used in this study are rabbit anti-ZCCHC11 (TUT4, Proteintech), rabbit anti-ZCCHC6 (TUT7, Proteintech), rabbit anti-PAPD4 (TENT2, Abcam), mouse anti-Flag (Sigma) and mouse anti- $\alpha$-tubulin (Sigma). The signals were developed with Pierce ECL plus Western Blotting Substrate (Pierce) and imaged by the Chemidoc Touch Imaging System (Bio-Rad).

\section{Immunoprecipitation for ectopically expressed TUT4 and TUT7 proteins}

pIRESneo-FLAG/HA-TUT4 or pIRESneo-FLAG/HA-TUT7 were transfected into HEK293T cells and cells were harvested in lysis buffer $(20 \mathrm{mM}$ Tris- $\mathrm{HCl}$ at $\mathrm{pH} 8.0,137 \mathrm{mM} \mathrm{NaCl}, 1 \mathrm{mM}$ EDTA, 1\% Triton x-100, 10\% Glycerol, $1.5 \mathrm{mM} \mathrm{MgCl2,} 5 \mathrm{mM}$ DTT) $48 \mathrm{~h}$ post-transfection. Protein was purified using anti-Flag M2 beads (Sigma) and resuspended in Buffer D (200 mM KCl, $10 \mathrm{mM}$ Tris-HCl [pH 8.0], 0.2 mM EDTA). The IP samples were confirmed by Western Blot analysis with anti-TUT4 (Proteintech), anti-TUT7 (Proteintech) and anti-Flag antibody (Sigma).

\section{In vitro uridylation}

miRNAs were synthesized by IDT and radio-labeled at the 5' end with T4 polynucleotide kinase (NEB) and $\left(\gamma^{-32} \mathrm{P}\right)$ ATP (Perkin Elmer) at $37^{\circ} \mathrm{C}$ for $30 \mathrm{~min} .{ }^{32} \mathrm{P}$ labeled miRNAs were purified by G25 columns (GE). In vitro uridylation reaction was performed in a total volume of $30 \mu$ in 3.2 $\mathrm{mM} \mathrm{MgCl2,} 1 \mathrm{mM}$ DTT, $6 \mathrm{nM}$ UTP, $\sim 0.6 \mathrm{nM} 5$ ' end labeled miRNA ( $\left.1 \times 10^{4} \mathrm{cpm}\right), 1.33 \mathrm{U} / \mu \mathrm{l}$ RNase inhibitor (NEB) and $15 \mu \mathrm{l}$ of immuno purified proteins on beads in Buffer D. The reaction mixture was incubated at $37^{\circ} \mathrm{C}$ for $15 \mathrm{~min} .10 \mu \mathrm{l}$ out of $30 \mu \mathrm{l}$ of the RNA was denatured and run on a $20 \%$ urea polyacrylamide gel. The sequences of miRNAs are listed in Table S2.

\section{Small RNA-seq library preparation}

Small RNA libraries were generated using QIAseq miRNA Library Kit (QIAGEN) according to the manufacturer instructions, except that the library DNAs were size-selected and purified by running in a native $6 \%(\mathrm{w} / \mathrm{v})$ acrylamide gel and followed by ethanol precipitation. Library quality was assessed using Qubit dsDNA HS Assay Kit (ThermoFisher) and Agilent High Sensitivity DNA kit (Agilent). Libraries were pooled together and sequenced on Illumina miSeq or NEXTseq platforms according to the manufacturer's specifications.

\section{MicroRNA expression and 3' isomiR analysis}

MiRNA expression levels and 3' isomiR composition were analyzed using QuagmiR on the $\mathrm{NCl}$ Cancer Genomics Cloud ${ }^{58}$. The classification between templated and non-templated (NT) isomiRs was assigned using genomic context obtained from miRBase v22 ${ }^{59}$. For those miRNAs that present multiple paralogs copies, all genomic loci were considered. More details of the $\mathrm{R}$ analysis are reported on GitHub (https://github.com/Gu-Lab-RBL-NCI/TUT-tailing). 


\section{Analysis of tail composition}

Annotations from miRBase v22 ${ }^{59}$ were used to classify miRNA isoforms as canonical, trimmed (shorter than the annotated mature miRNA sequence), and tailed (longer than the annotated mature miRNA sequence). Tailed isoforms were classified into templated and non-templated as previously described. Moreover, un-templated tailing events were subdivided into three major categories: mono-adenylation, mono-uridylation, and mixed (containing all other mono-tailing and oligo-tailing possibilities). More details of the $\mathrm{R}$ analysis are reported on GitHub (https://github.com/Gu-Lab-RBL-NCI/TUT-tailing/tree/main/processing\%20miRNA).

\section{Tailing cumulative curves}

The global impact of knockout and rescue of TENT2/TUT4/TUT7 on tailing was measured using cumulative curves. Briefly, for each miRNA isoform a fold-change of abundance was calculated between the two experimental conditions. The resulting distribution of fold-changes obtained was sorted into bins representing the fraction of isomiRs with fold-changes equal or smaller than the set threshold value for that bin. More details of the $\mathrm{R}$ analysis are reported on GitHub (https://github.com/Gu-Lab-RBL-NCI/TUT-tailing/tree/main/cumulative-curves-tailing).

\section{Statistical analysis}

The Wilcoxon test was used to determine $p$-values, and values $<0.05$ were considered statistically significant. Statistical analysis was done in RStudio and GraphPad Prism7 statistical software.

\section{Data availability}

Small RNA-seq data is deposited at GEO with the accession number GSE183384 and GSE184550.

\section{Figure Legends}

\section{Fig. 1 miRNA 3' tailing is frequent and specific}

Analyses of the top 200 most expressed miRNAs (top $1005 p$ and top $1003 p$ miRNAs). a Pie chart on the 3' end composition (percentages). b Nucleotide identity on NT tails (average percentage \pm standard error). c Nucleotide identity on NT tails and tail length. Column height represents the percentage of NT tail while colored areas represent the corresponding fraction based on nucleotide identity. U_tail and A_tail represent uridylation and adenylation homogenous tailing, whereas Mixed includes heterogeneous tailing and other nucleotides. d Percentage of NT isomiRs for the 10 least and most tailed miRNAs. e Examples of isomiR composition for miRNAs with the least (miR-16-5p) and the most (miR-345-5p) NT tailing.

\section{Fig. 2 Coordination of TENT2, TUT4 and TUT7 in miRNA 3' tailing.}


a Two clones were used for each isogenic cell line. TENT2, TUT4 and TUT7 were detected by Western blot. Asterisks indicate non-specific bands. b Nucleotide identity on NT tails in the different knockout clones on the top 200 most expressed miRNAs. Column height represents the percentage of NT tail while colored areas represent the corresponding fraction based on nucleotide identity. c Box-plot of the average length change (delta) between the different knockouts and WT cells. Average lengths were weighted based on relative isomiR abundances within each miRNA. d miR-345-5p, miR-24-3p, and miR-16-5p in each isogenic cell line were detected by Northern blot.

Fig. 3 TENT2 contributes to adenylation, uridylation, and guanylation of miRNAs.

a Scheme of the experimental strategy. b Heatmaps of the relative abundance of mono-tailed isomiRs (sequences are indicated in square brackets) between WT and knockout cells. Colorimetric scale ranges in values between 0 and $\geq 100$. c Cumulative curves of all mono-tailed isomiRs upon knockout or rescue of TENT2 in different cellular knockout backgrounds. Colored lines indicate canonical miRNAs (black), templated/ambiguous (green) and NT (blue) isomiRs. d Scheme of the nucleotide positions analyzed. Estimated $A$ to $G$ conversions were calculated based on the known " $A$ " identity of the sequence on synthetic or endogenous miRNAs (columns 1 to 3 ) and benchmarked against the observed abundance of NT A and G tails (column 4).

Fig. 4 TENT2 selectively modifies mature miRNAs but has minimal impact on miRNA abundance.

a Percentage of adenylated isomiRs across different knockouts and rescues. Sequences indicate templated nucleotides (black) and NT adenylation (red). b-c Scatter plot of fold-change in the percentage of NT A-tail between different genetic backgrounds and rescues. $\mathbf{d}$ Scatter plot of fold-change in the percentage of NT A-tail and U-tail (matched isomiRs) between different genetic backgrounds. $R$ was calculated using the Pearson correlation coefficient. e Scatter plot of fold-changes in the percentage of NT A-tail (x-axis) and overall miRNA abundance (y-axis) upon TENT2-KO. f-g Scatter plot of fold-changes in overall miRNA abundance upon TENT2 knockout or rescue in different genetic backgrounds.

\section{Fig. 5 TUT4 but not TUT7 selectively uridylates most mature miRNAs.}

a Heatmap of the relative abundance of mono-uridylated isomiRs between WT and knockout cells. Colorimetric scale ranges in values between 0 and $\geq 100$. $\mathbf{b}$ Cumulative curves of all mono-uridylated (upper panel) and mono-adenylated (lower panel) isomiRs upon knockout of TUT4 or TUT7. Colored lines indicate canonical miRNAs (black), templated/ambiguous (green) and NT (blue) isomiRs. c Percentage of uridylated isomiRs across single and double knockout of TUT4 and TUT7. Sequences indicate templated nucleotides (black) and NT adenylation (red). d Scatter plot of fold-changes in the percentage of NT U-tail upon the knockout of TUT4 in different genetic backgrounds. Dots highlighted in blue indicate a subset of uridylated isomiRs more sensitive to TUT4. e Analysis of TUT4 tailing preference based on the last templated nucleotide preceding the uridylation. The TUT4-sensitive group $(\mathrm{N}=100)$ was defined based on the TUT4 knockout response described in Fig. $5 \mathrm{~d}$. $\mathrm{f}$ In vitro uridylation of single stranded miRNA 
by purified TUT4 or TUT7 protein. $\mathbf{g}$ In vitro uridylation of single stranded miRNA where the last nucleotide has been changed from " $\mathrm{G}$ " to " $\mathrm{C}$ ".

Fig. 6 TUT4/7 regulate the abundance of a set of miRNAs via distinct mechanisms.

a Heatmap illustrating the miRNAs with the highest expression increase upon TENT2, TUT4 and TUT7 knockouts. b Analysis comparing the effects on miRNA abundance upon knockout and rescue with TUT4, TUT7 or TUT4/7 in different genetic backgrounds. Colored dots indicate $\log 2(\mathrm{FC}) \geqslant 0.2$ (upregulation, red) and $\log 2(\mathrm{FC}) \leqslant 0.2$ (downregulation, blue). Dashed circle indicates members of the miR-888 cluster. c Venn diagram illustrating miRNAs positively (left panel) and negatively (right panel) regulated by TUT4/7. This diagram was based on the consensus responses observed in Fig. 6b. d Northern blot validating the level changes of TUT4 and TUT7 regulated miRNAs in WT, DKO and TKO cells. e Northern blot validating TUT4 and TUT7 rescue effects on the levels of TUT4 and TUT7 regulated miRNAs. $f$ Northern blot detecting miRNA levels in WT, DKO, and TKO cells transfected with control or LIN28B siRNA. $\mathbf{g}$ The levels of miR-888 and miR-892a in the DKO and TKO cells upon rescue with TUT7-WT or TUT7-CD were detected by Northern blot, Let-7i served as a control. 
a

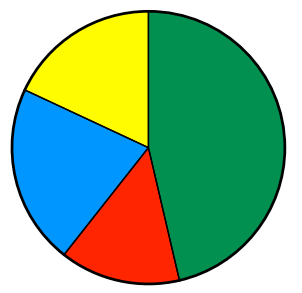

5p miRNAs

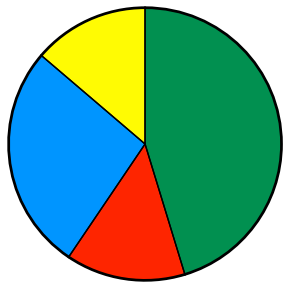

All miRNAs

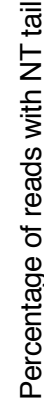

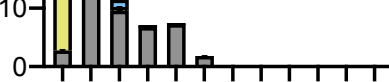

123456789101112

Length non-templated tail $\square$ Canonical

$\square$ Trimmed

Templated tail

Non-templated tail

\section{All miRNAs}

3p miRNAs

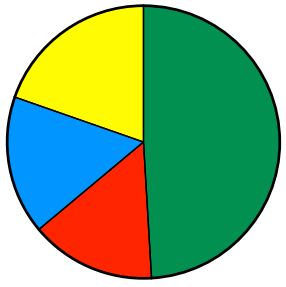

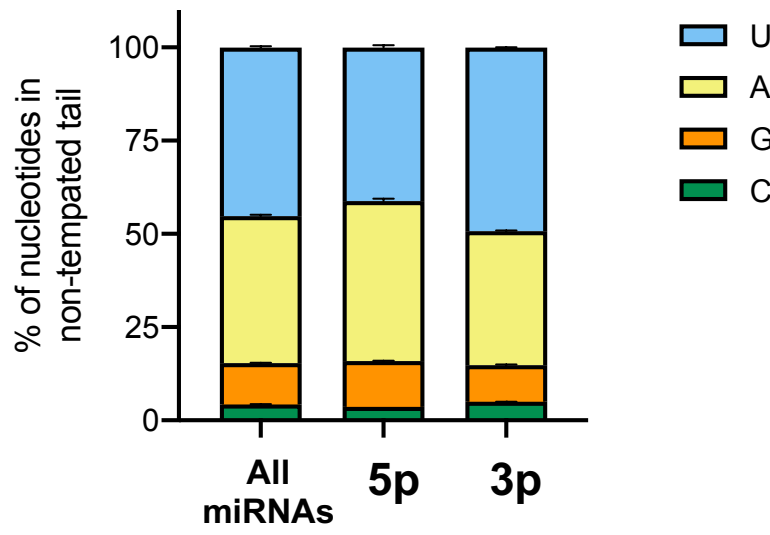

5p miRNAs

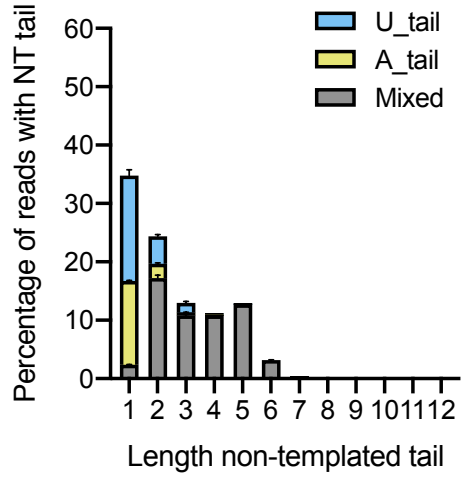

3p miRNAs

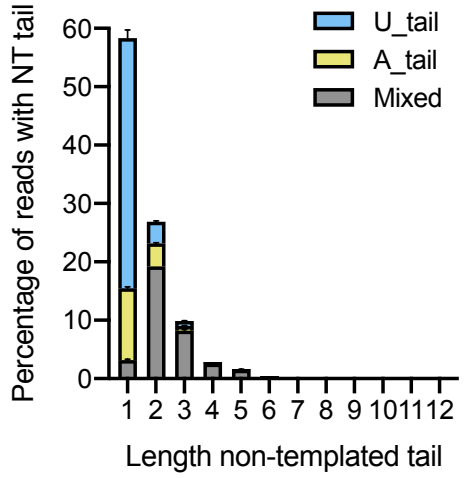

d

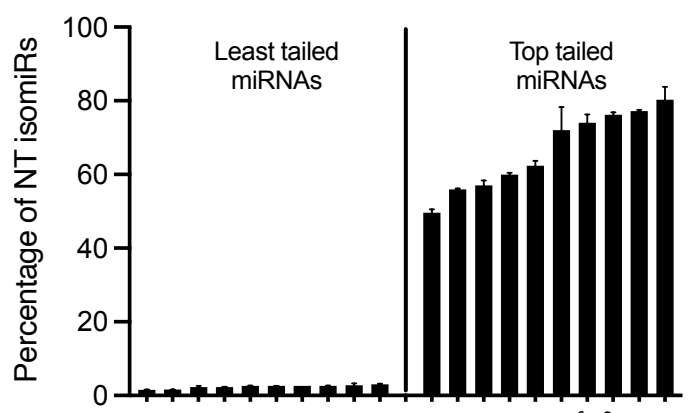

$\mathbf{e}$

miR-16-5p

\begin{tabular}{c|cc} 
UAGCAGCACGUAAAUAUUGGCG & Canonical & $88.3 \%$ \\
UAGCAGCACGUAAAUAUUGGCGU & Templated tailing & $4.4 \%$ \\
UAGCAGCACGUAAAUAUUGGC & Trimmed & $0.7 \%$ \\
UAGCAGCACGUAAAUAUUGGCGA & Non-templated tailing & $0.5 \%$ \\
$\ldots$ & & \\
... & Genomic sequence
\end{tabular}

...YYTAGCAGCACGTAAATATTGGCGTW... Genomic sequence

\begin{tabular}{|c|c|c|}
\hline \multicolumn{3}{|c|}{ miR-345-5p } \\
\hline GCUGACUCCUAGUCCAGGGCUC: & Canonical & $4.7 \%$ \\
\hline GCUGACUCCUAGUCCAGGGCU & Trimmed & $8.4 \%$ \\
\hline CUGACUCCUAGUCCAGGGCUCU & Non-templated tailing & $56.0 \%$ \\
\hline CUGACUCCUAGUCCAGGGCUCA & Non-templated tailing & $2.4 \%$ \\
\hline
\end{tabular}


a

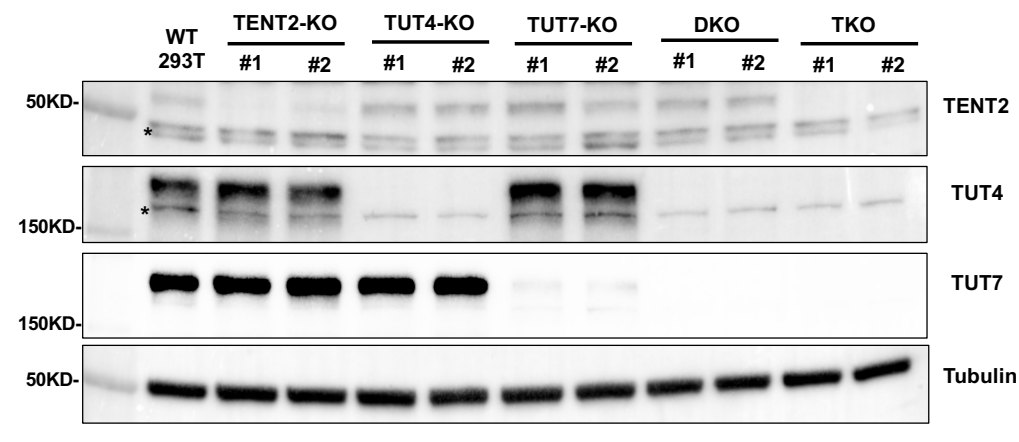

b

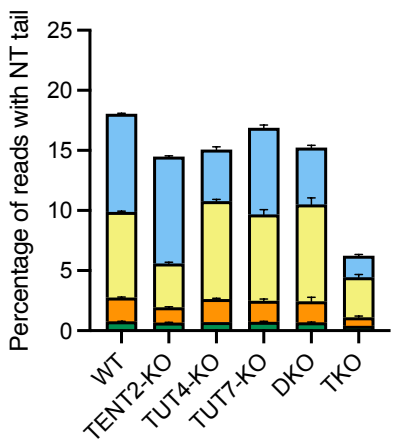

C

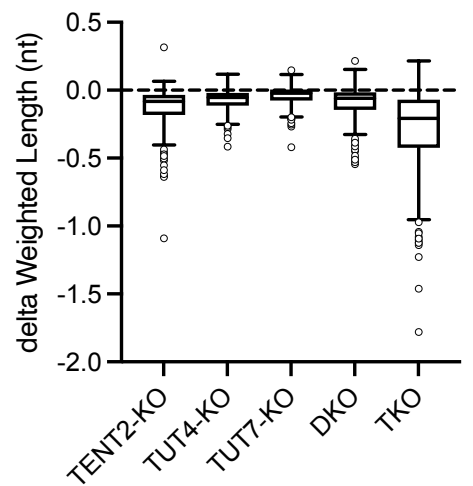

O W $\frac{\text { WT HEK293T }}{\# 1 \quad \# 2} \frac{\text { TENT2-KO }}{\# 1 \quad \# 2} \frac{\text { TUT4-KO }}{\# 1 \quad \# 2} \frac{\text { TUT7-KO }}{\# 1 \quad \# 2} \frac{\text { DKO }}{\# 1 \quad \# 2} \frac{\text { TKO }}{\# 1 \quad \# 2}$

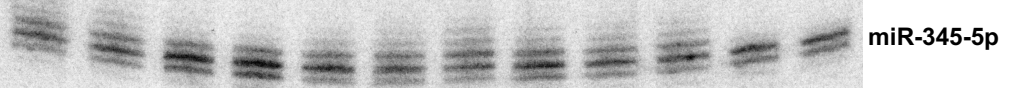

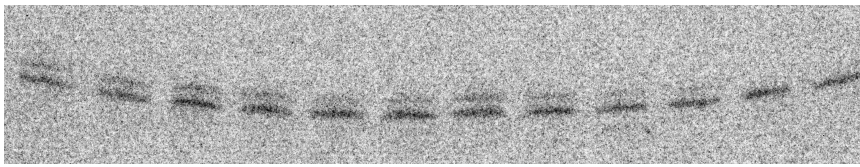

miR-24-3p

miR-16-5p 


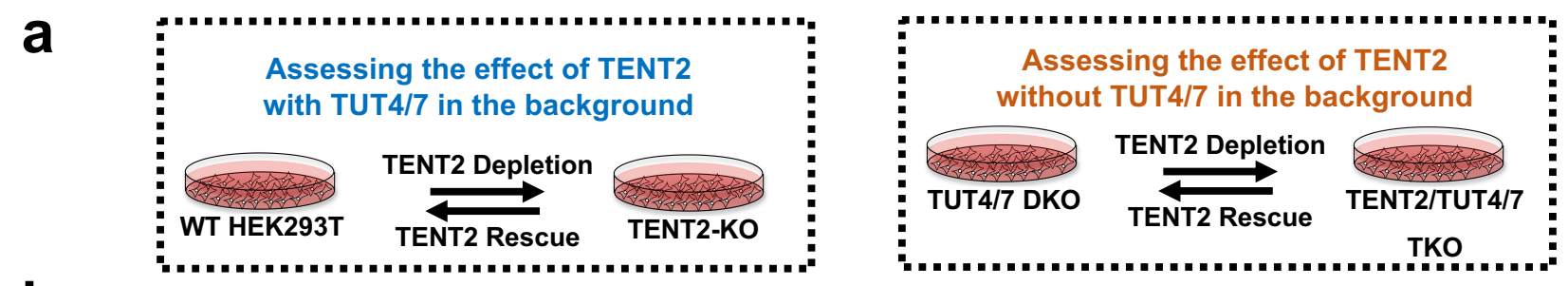

b

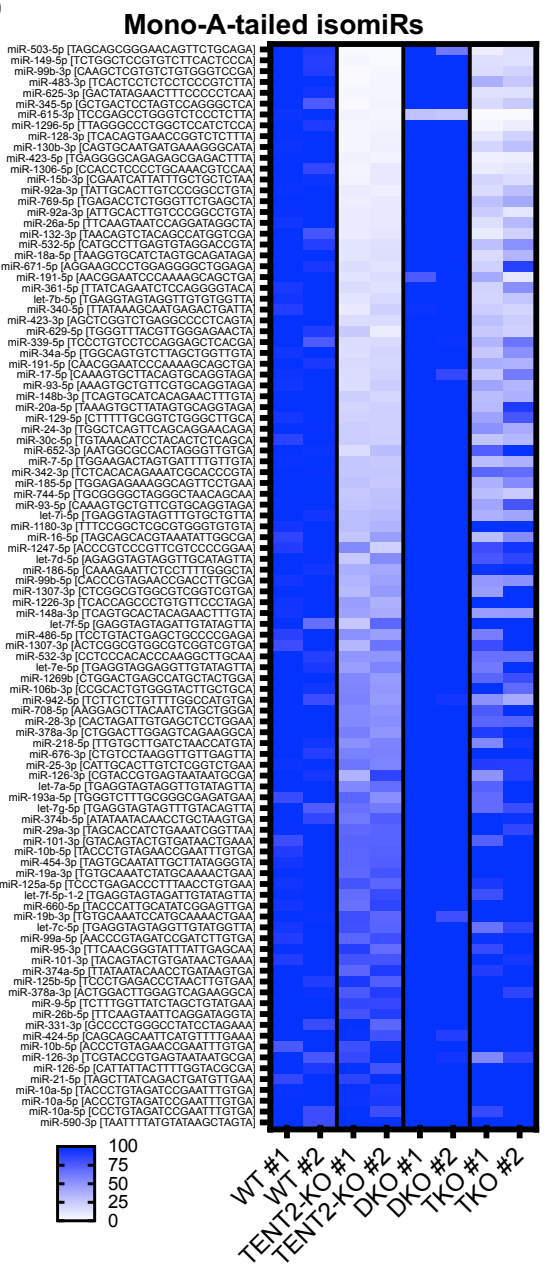

C

(5)

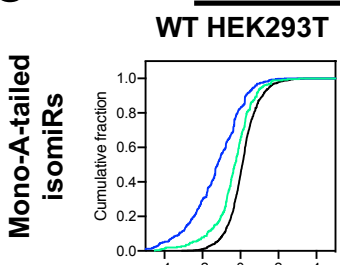

$\log _{2}$ FC (CPM) TENT2-KO/WT

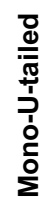



ס

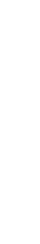

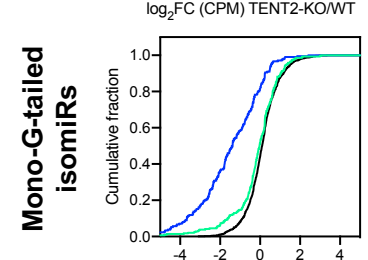

$\log _{2} \mathrm{FC}$ (CPM) TENT2-KO/M

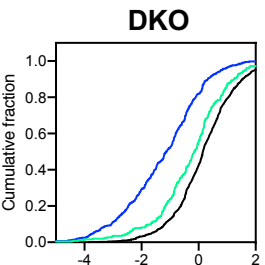

$\log 2$ FC (CPM) TKO/DKO

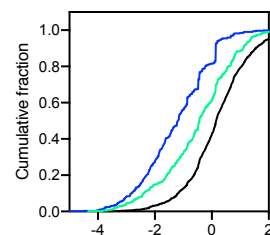

$\log 2$ FC (CPM) TKO/DKO

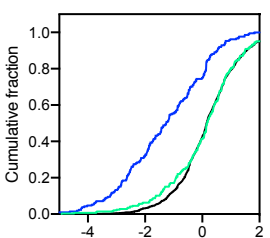

$\log 2 \mathrm{FC}$ (CPM) TKO/DKO

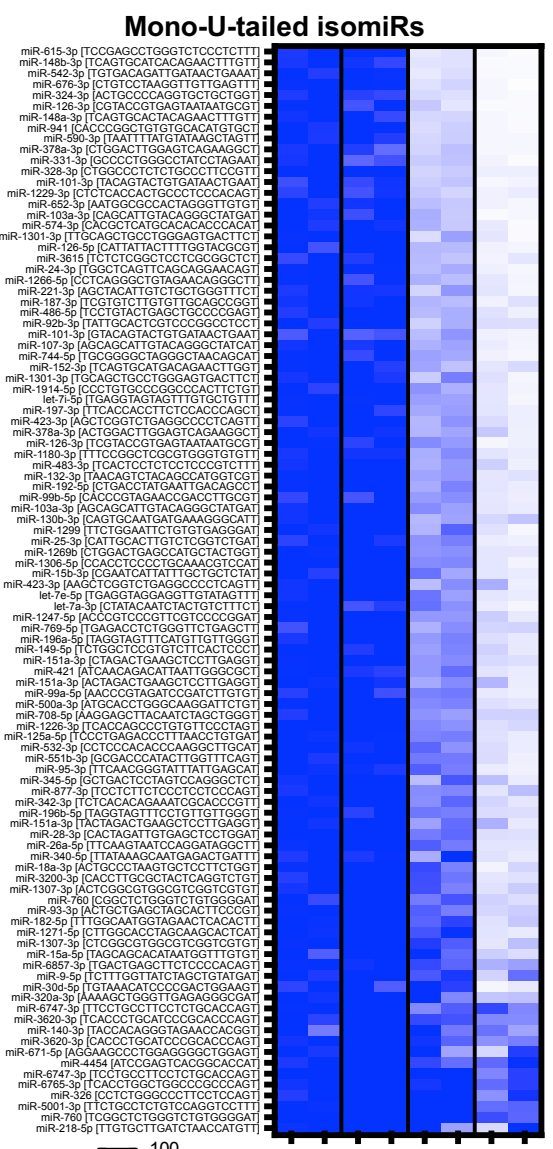

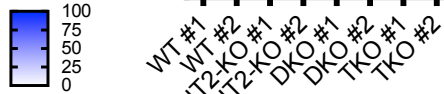
退金

Rescue with TENT2 on

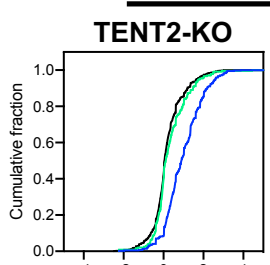

TKO

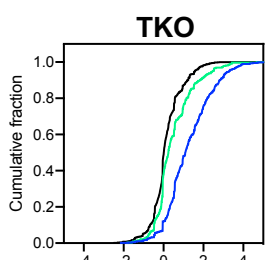

$\log _{2}$ FC (CPM) TENT2/GFP rescue $\log _{2}$ FC (CPM) TENT2/GFP rescue
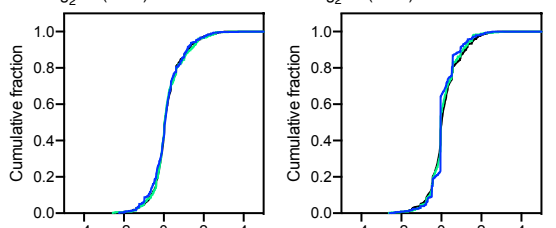

$\log _{2}$ FC (CPM) TENT2/GFP rescue $\log _{2}$ FC (CPM) TENT2/GFP rescue

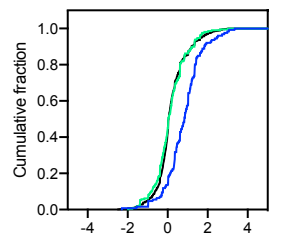

$\log _{2} \mathrm{FC}$ (CPM) TENT2/GFP rescue $\log _{2}$ FC (CPM) TENT2/GFP rescue

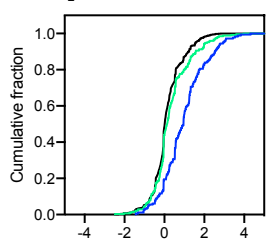

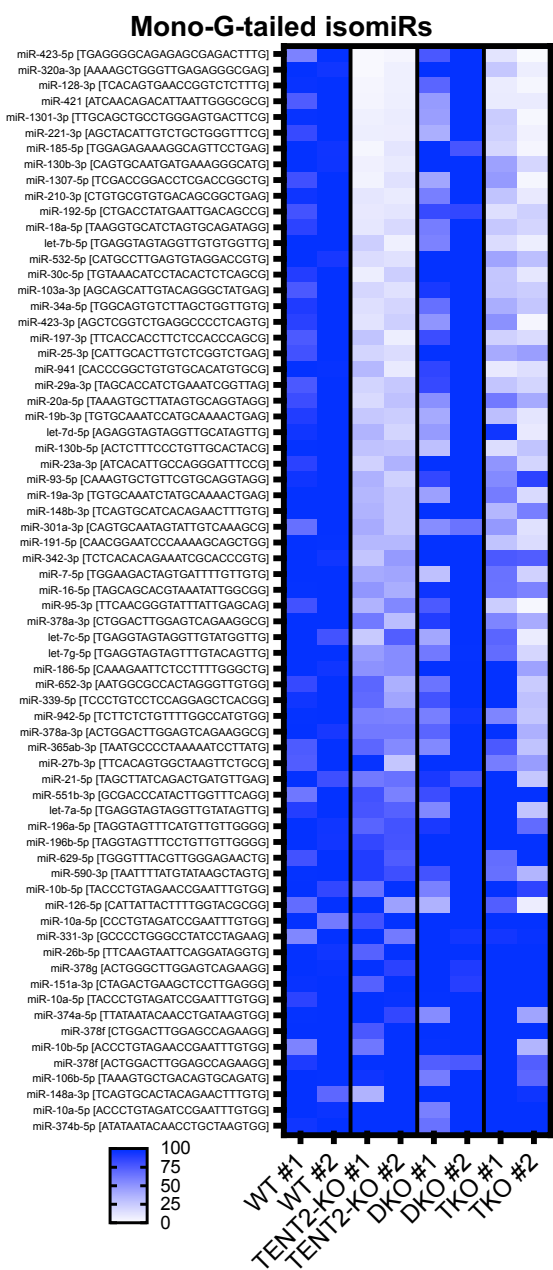

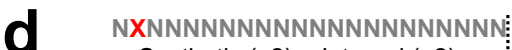
Synthetic (g2) + Internal (g2)

NNNNNNNNNNNNNNNNNNNNNX Last nucleotide

NNNNNNNNNNNNNNNNNNNNNN Non-templated tail

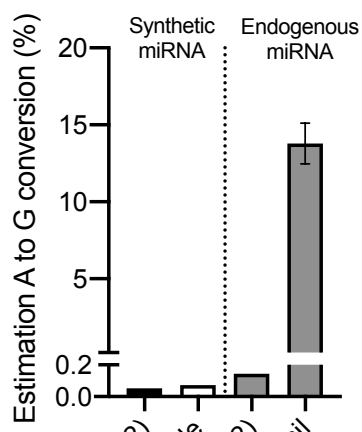


a

miR-615-3p
TCCGAGCCTGGGTCTCCCTCTA miR-10a-5p

AGATCCGAATTTGTGA

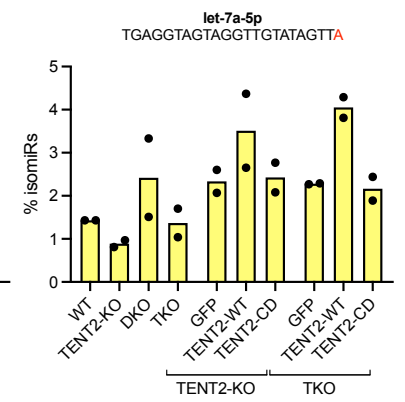

C
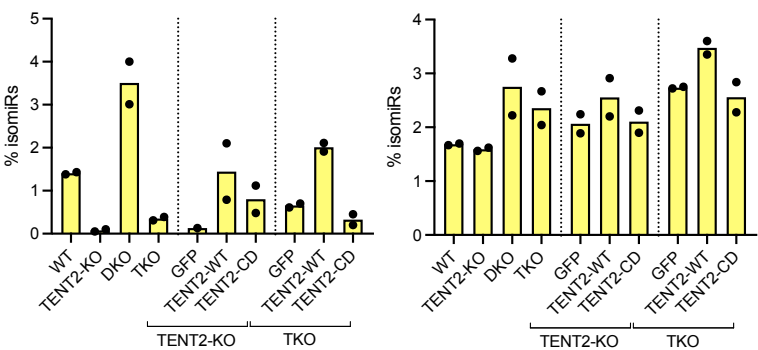

TENT2-mediated adenylation

With TUT4/7 in the background

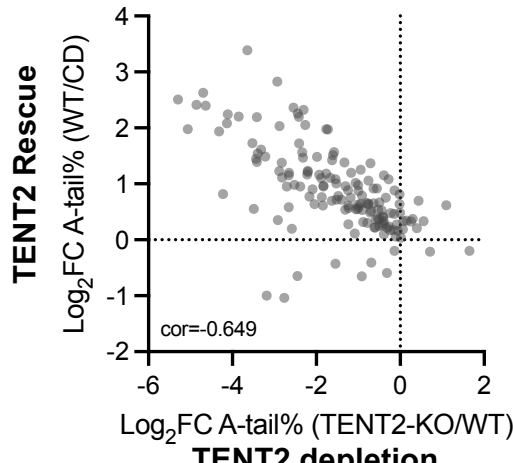

TENT2 depletion

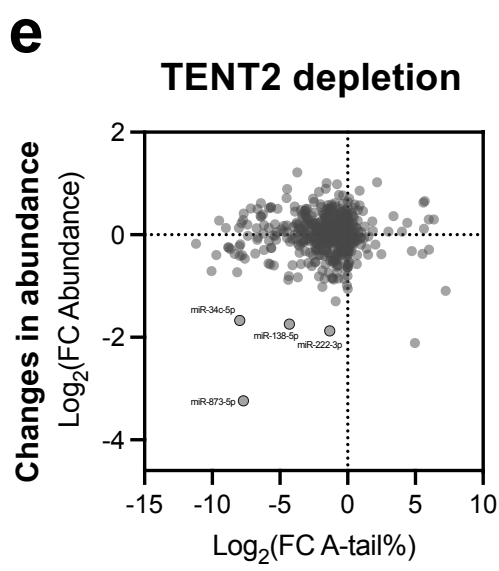

Changes in adenylation
Without TUT4/7 in the background

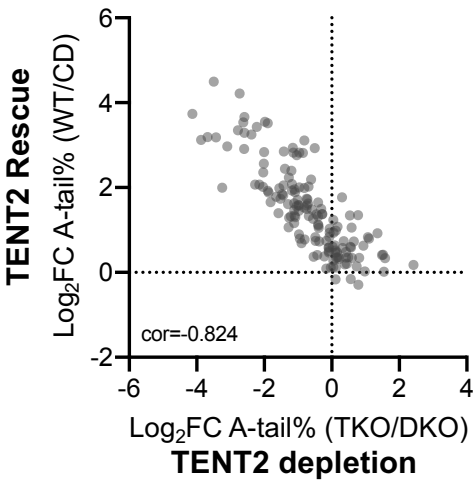

f

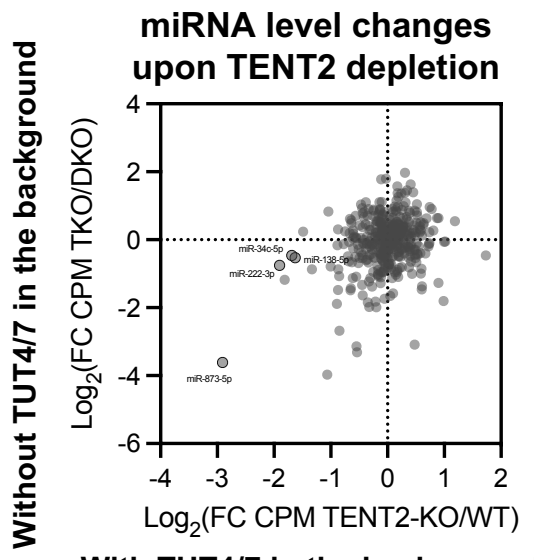

With TUT4/7 in the background b
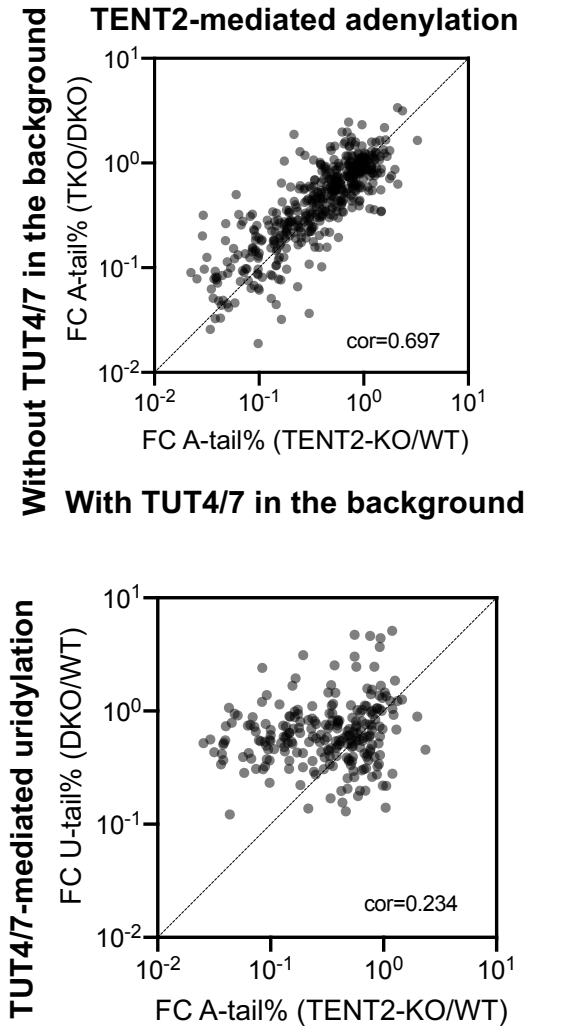

TENT2-mediated adenylation

g

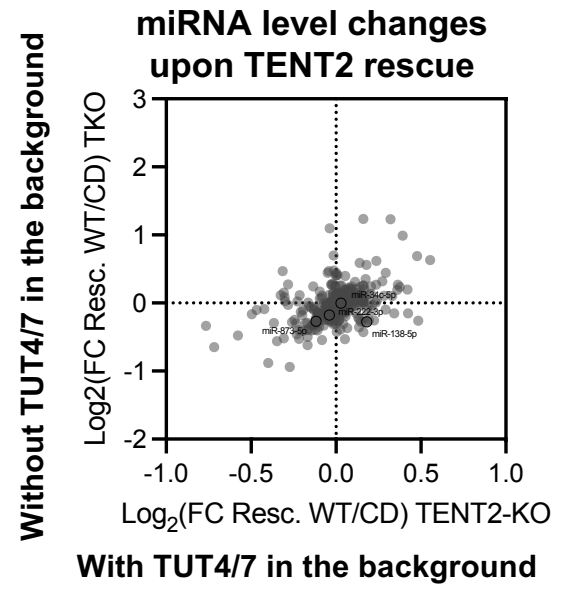



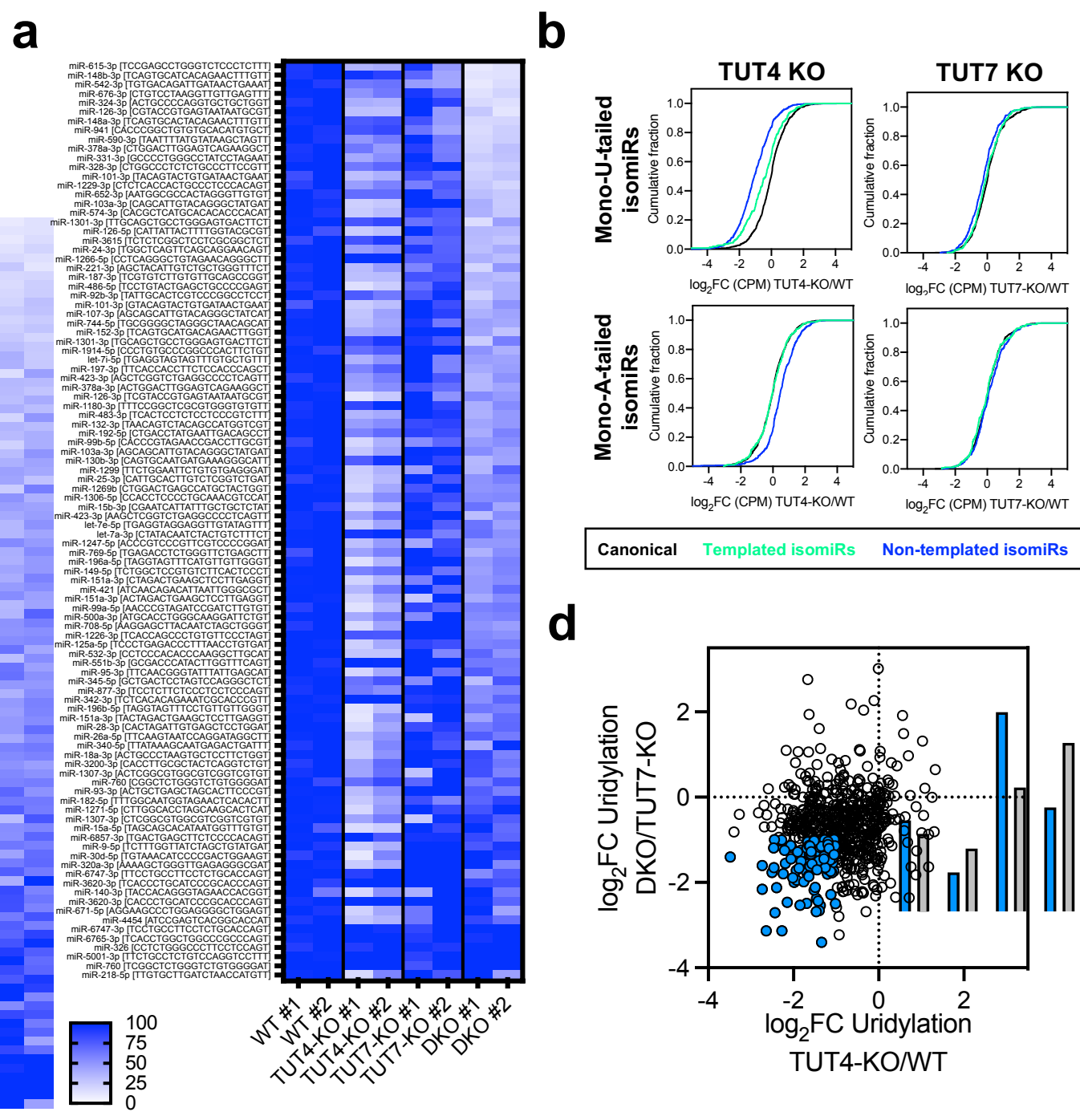

C
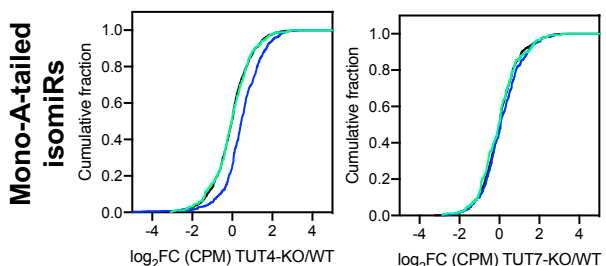

Canonical Templated isomiRs Non-templated isomiRs
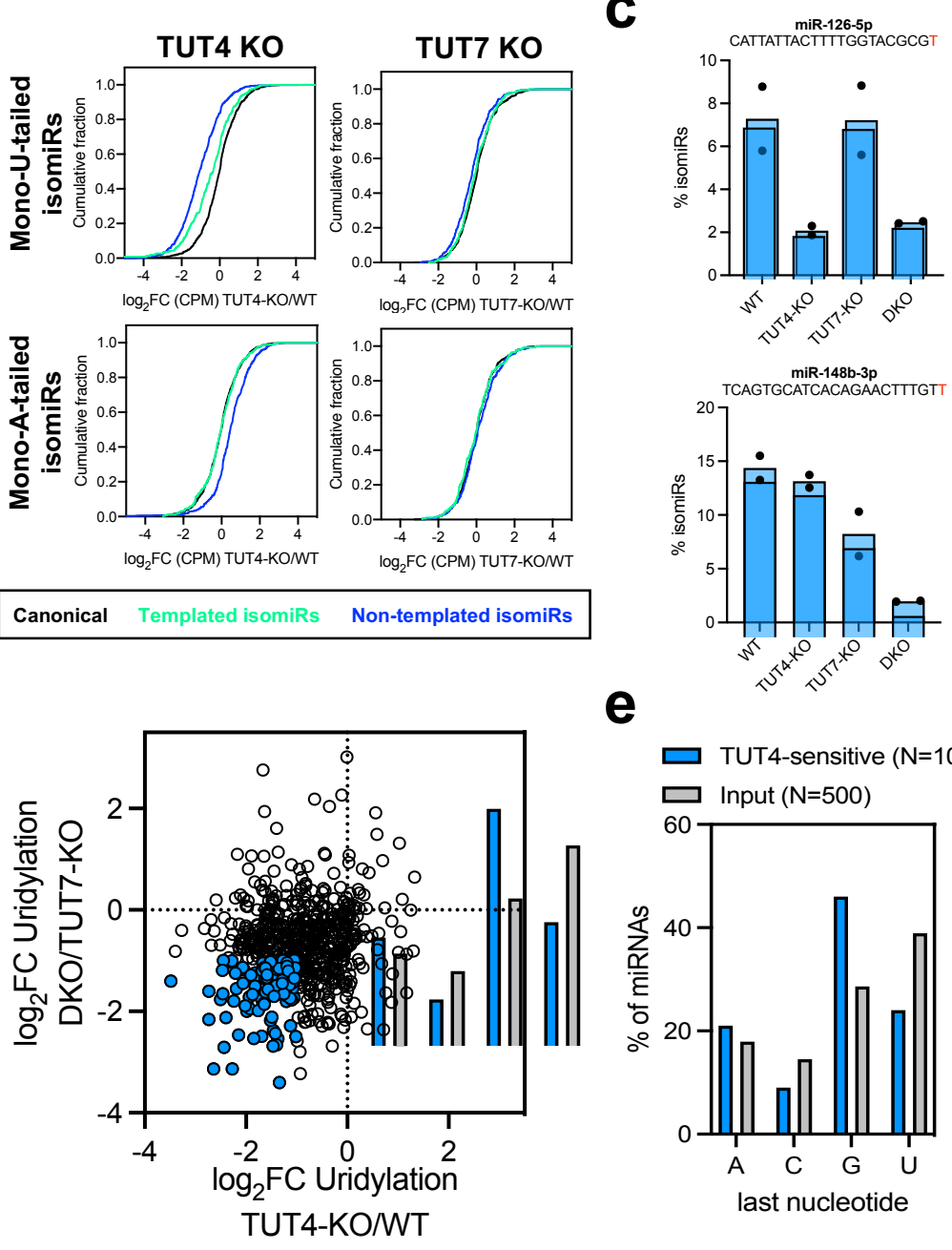

e

$\square$ TUT4-sensitive $(\mathrm{N}=100)$

$\square$ Input $(\mathrm{N}=500)$

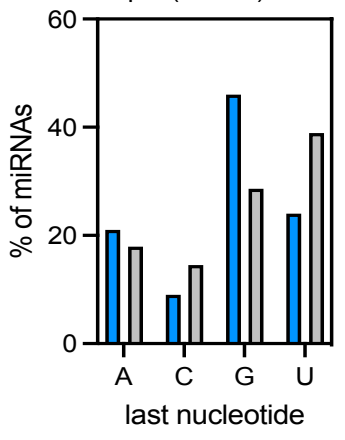

f

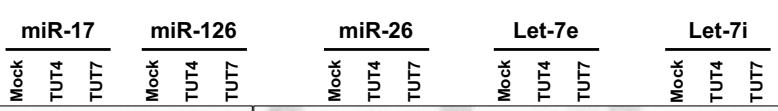

g

\begin{tabular}{|c|c|c|c|c|c|}
\hline \multicolumn{3}{|c|}{ miR-17 } & \multicolumn{3}{|c|}{ miR-126 } \\
\hline lock & TUT4 & TUT7 & Mock & TUT4 & TUT7 \\
\hline C & G C & & G C & $\overline{G \quad C}$ & G \\
\hline
\end{tabular}

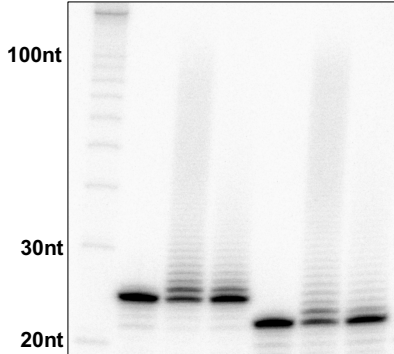




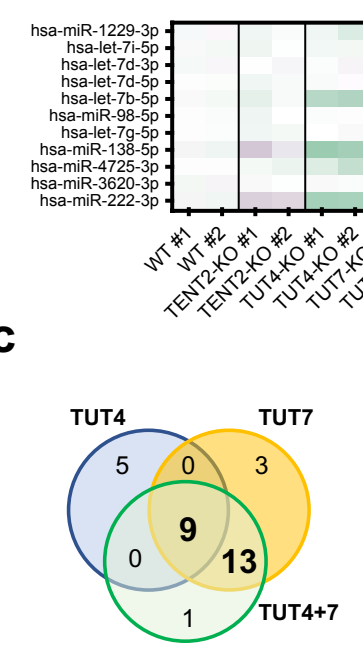

miRNAs positively regulated by TUT4/7

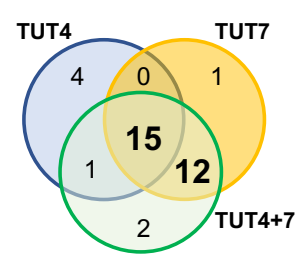
miRNAs negatively regulated
by TUT4/7
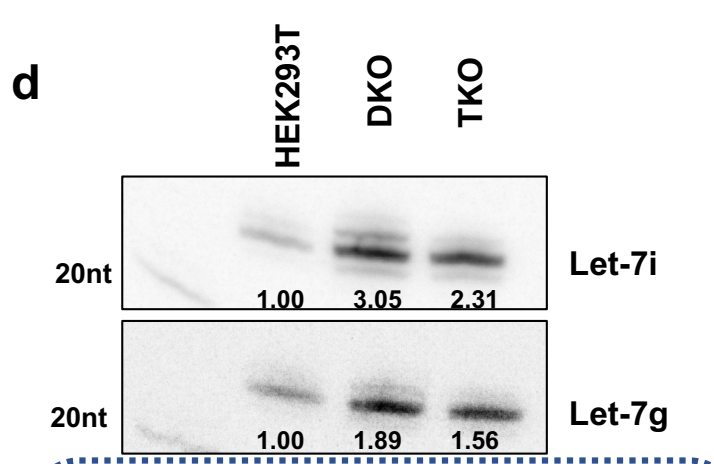

Let-7g
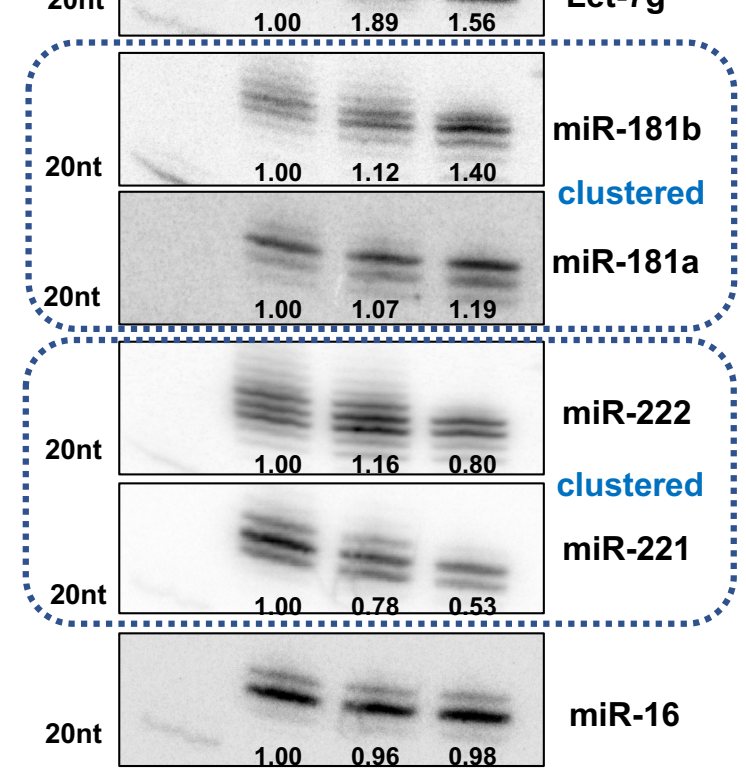

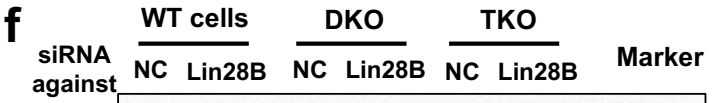

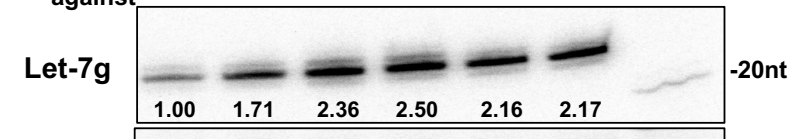

miR-181b

$\log _{2}$ (FC DKO/WT)
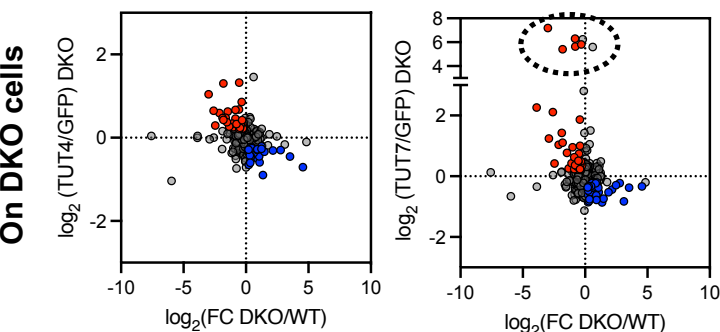

$\log _{2}$ (FC DKO/WT)
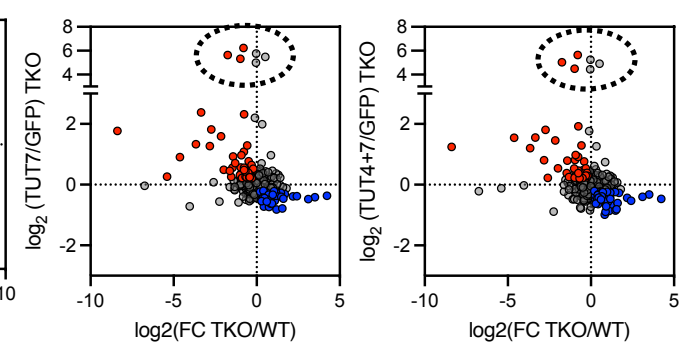

$\log _{2}$ (FC DKONT)

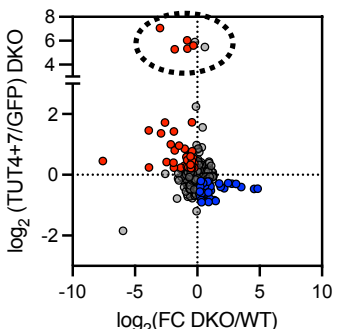

cells
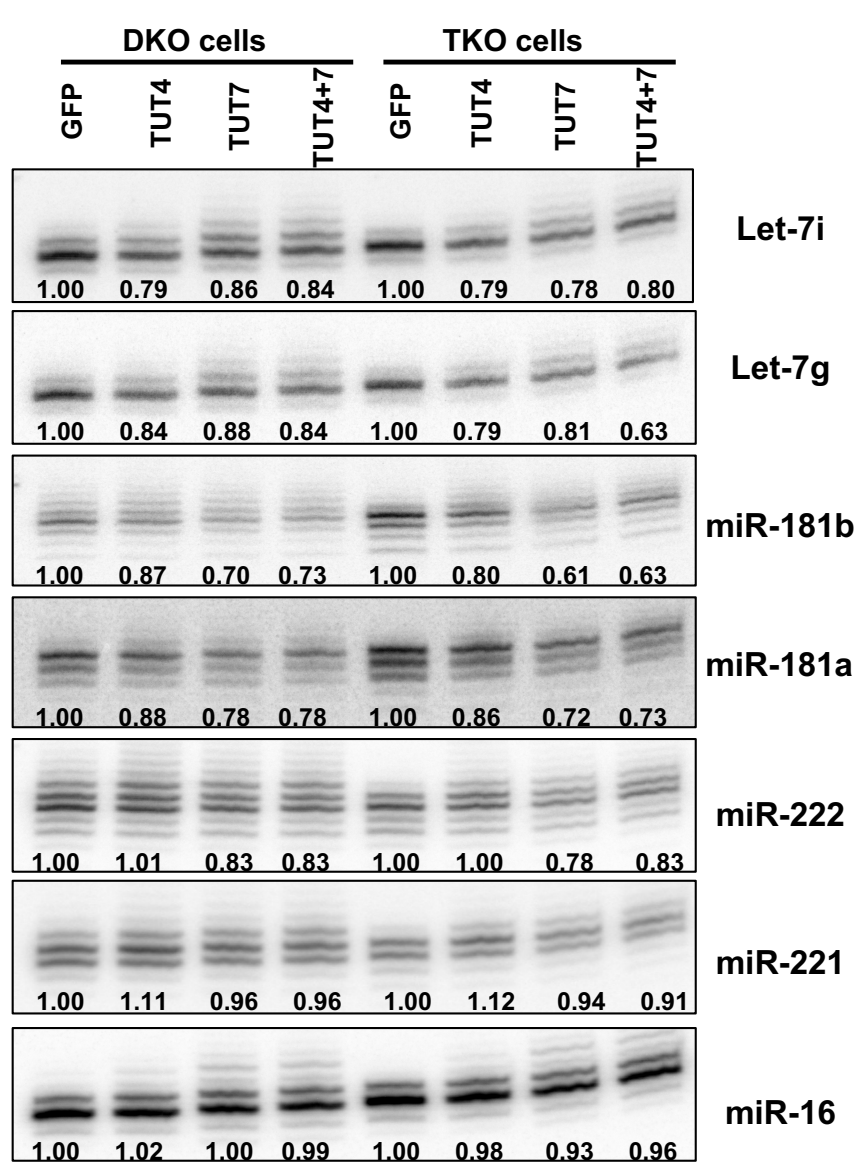

miR-221

miR-16

20nt

miR-888

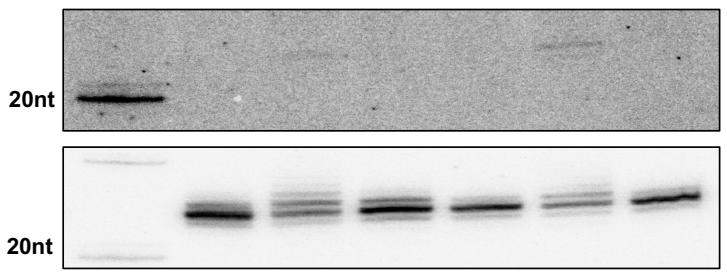

miR-892a

Let-7i 